\title{
Predictors of disease severity and outcome of hospitalized renal transplant recipients with COVID-19 infection: a systematic review of a globally representative sample
}

\author{
IRTIZA HASAN ${ }^{1,3}$, TASNUVA RASHID ${ }^{2}$, SARAH SULIMAN $^{3}$, HATEM AMER $^{4}$, RAZVAN M. CHIRILA ${ }^{5}$, \\ MARTIN L. $\mathrm{MAI}^{3}$, TAMBI JARMI ${ }^{3}$, SAMIR KHOUZAM ${ }^{3}$, PABLO MORENO FRANCO ${ }^{3}$, \\ CHARLES W. HEILIG ${ }^{1}$, HANI M. WADEI ${ }^{3}$

\footnotetext{
${ }^{1}$ Department of Medicine, Division of Nephrology, University of Florida College of Medicine, Jacksonville, FL

${ }^{2}$ The University of Texas School of Public Health, Houston, TX

${ }^{3}$ Department of Transplant, Mayo Clinic, Jacksonville, FL

${ }^{4}$ William J von Liebig Center for Transplantation and Clinical Regeneration, Mayo Clinic, Rochester, Minnesota
} \\ ${ }^{5}$ Department of Medicine, Mayo Clinic, Jacksonville, FL
}

\begin{abstract}
Introduction. COVID-19 presents a special challenge to the kidney transplant population.
Methods. A systematic review of articles that examined COVID-19 in kidney transplant recipients was performed. Patients' demographics, clinical, laboratory and radiological presentations, immunosuppression modification, and COVID-19 specific management were abstracted and analyzed. COVID-19 severity was classified into mild, moderate, and severe. Disease outcome was classified by whether the patient was discharged, still hospitalized, or died.

Results. 44 articles reporting individual data and 13 articles reporting aggregated data on 149 and 561 kidney transplant recipients respectively with COVID-19 from Asia, Europe and America fulfilled all inclusion and exclusion criteria. Among studies reporting case specific data, $76 \%$ of cases had severe disease. Compared to patients with mild/moderate disease, patients with severe disease had higher CRP, LDH, Ferritin, D-dimer and were more likely to have bilateral lung involvement at presentation and longer time since transplantation $(\mathrm{P}<0.05$ for all). Recipients' age, gender and comorbidities did not impact disease severity. Patients with severe disease had a more aggressive CNI reduction and more antiviral medications utilization. Outcome was reported on 145 cases, of those 34 $(23 \%)$ died all with severe disease. Longer duration from transplant to disease diagnosis, hypoxia and higher LDH were associated with mortality $(\mathrm{P}<0.05)$. Different immunosuppression reduction strategies, high dose parenteral corticosteroids use and various antiviral combinations did not demonstrate survival advantage. Similar finding was observed for studies reporting aggregated data.

Conclusion. COVID-19 in kidney transplant patients is associated with high rate of disease severity and fatality. Higher $\mathrm{LDH}$ and longer time since transplantation predicted both disease severity and mortality. None of the COVID-19 specific treatment correlated with, or improved disease outcome in kidney transplant recipients.
\end{abstract}

Key words: coronavirus disease 2019, COVID-19, Severe Acute Respiratory Syndrome Coronavirus 2, SARS-CoV-2, novel coronavirus; coronavirus pandemic, renal transplant, transplant recipient, immunosuppression.

\section{INTRODUCTION}

Coronavirus disease 2019 (COVID-19) is a novel, highly contagious, and pathogenic disease caused by Severe Acute Respiratory Syndrome Coronavirus 2 (SARS-CoV-2) and responsible for the current global pandemic outbreak which took the health care community by surprise [1]. Although COVID-19 was introduced to the human population from the animal kingdom, the spread in the human population has been entirely driven by human to human transmission $[2,3]$. Transmission is primarily through droplets or direct contact but indirect contact through contaminated surfaces has also been documented [4]. In host cells, the SARS-CoV-2 virus binds to host cell angiotensin converting enzyme 2 (ACE2) receptors which are highly expressed in the lung epithelium, heart, ileum, kidney, and bladder [5]. The interaction of virus affected cells with host immune systems namely innate and adaptive immune cells results in a constellation of symptoms [4]. In the general population, there are 3 major patterns of clinical illness with mild flu-like symptoms, non-life-threatening pneumonia, or severe pneumonia progressing to adult respiratory distress syndrome (ARDS) requiring advanced life support $[3,6]$. Other systems involvement like cardiovascular, neurological, renal, and gastrointestinal, have also been commonly 
reported [7]. Cytokine storm syndrome, secondary hemophagocytic lymphohistiocytosis, and coagulation disorders have also been reported and are some of the determinants of disease severity [3]. Recently, the World Health Organization (WHO) classified COVID-19 severity into mild, moderate and severe according to symptomatology and clinical presentations $[8,9]$.

Kidney transplant recipients are prone to be infected by opportunistic pathogens and hence can be assumed to be one of the most vulnerable groups in the COVID-19 pandemic. Although the true impact of COVID-19 on transplant recipients is mostly unknown, it is imperative to anticipate the potential consequences of COVID-19 in this at-risk population. Kidney transplant recipients with COVID-19 pose unique challenges, including the potential for rapid clinical progression, risk of increased mortality, and need to manage immunosuppressive medications with limited data, possibility of drug-drug interaction, and short and long term risk of allograft rejection or loss. With the start of the COVID-19 pandemic, multiple case reports and case series emerged from around the globe describing: the clinical presentation; anecdotal immunosuppression management; course of kidney transplant and other solid organ transplant recipients. These reports showed wide variation in the reported disease severity, immunosuppression modification and changes, antiviral treatment and outcome which left the transplant community without reasonable direction on how best to manage kidney transplant patients with COVID-19. With the current expectation that the pandemic might be more prolonged than initially anticipated, we need guidance, extensive research, and critical evaluation of available evidence and risk-benefit calculations on how to treat COVID-19 in kidney transplant recipients. In this article, we sought to review the available evidence using a systematic approach that included all reported COVID-19 cases in kidney transplant recipients that required hospitalization. We aimed to do a systematic review of COVID-19 in renal transplant recipients to identify factors associated with disease severity and mortality with special emphasizes on immunosuppression and virus-specific management plans.

\section{MATERIAL AND METHODS}

We performed a systematic review to assess the evidence regarding predictors of COVID-19 severity and outcome in kidney transplant recipients. Studies that (i) examined COVID-19 in kidney transplant recipients, (ii) provided case-specific or aggregate information for renal transplant recipients, (iii) were published in the English language, and (iv) in the adult population with (v) available full texts were included in the review. Studies were excluded if they (i) were not related to COVID-19; (ii) looked at any coronavirus other than novel coronavirus sars 2; (iii) were laboratory, genetic or bench studies; (iv) were not original studies; (v) looked at transplant patients other than kidney transplant recipients; (vi) did not include management strategies for COVID-19 in kidney transplant recipients or (vii) looked at infection control strategies and (viii) did not include individual or aggregate data for renal transplant recipients alone or did not include outcome for hospitalized patients. We systematically searched for articles indexed in PubMed (NLM) and Medline (Ovid) using keywords and mesh terms with the last date of search being July 16, 2020. Concepts used in the search included SARS-COV-2, COVID-19 and renal transplant. Search strategy for PubMed database is provided in supplementary Table 1. Bibliographies of selected articles were searched for additional studies not identified through our initial database search. All abstracts and full texts were independently reviewed by two authors and any discrepancies resolved by consensus. RefWorks [ProQuest, Ann Arbor, MI] was used as a citation manager for the removal of internal and external duplicates among the databases. Two authors independently reviewed and abstracted information and findings tabulated into descriptive evidence tables. Separate evidence tables were created for studies providing individual data as well as for those providing aggregate data for capturing maximum information. Data collected included country of origin, time of publication, patient demographics and comorbidities, transplant information, baseline renal function, clinical symptoms, diagnosis of COVID-19, significant laboratory values, imaging studies, baseline and change in immunosuppression, COVID-19 specific treatment, ICU admission and intubation and clinical outcome. The risk of bias and clinical heterogeneity for included observational studies were appraised in two ways - firstly, setting strict inclusion and exclusion criteria for selection of articles and secondly, through quality assessment tool published by the National Institute of Health (NIH). Risk of bias was independently assessed by the two authors and any disagreement resolved by a third author. As COVID-19 is a new venture with daily upcoming new information, maximum attempt was made to collect and include majority of data for a full-fledged review. Quality control was assured and 
accomplished by (i) independent search of database by two authors; (ii) independent search of all titles and abstracts by two authors; (iii) screeners blinded to journal name and authors and (iv) independent review of all selected full-text articles by two authors. An MS Excel workbook (Microsoft Corp, Redmond, WA) was used to enter the data from different selected articles. Tests were done to compare the abstracted information from the articles by disease severity and outcome. COVID-19 severity was classified as per the World Health Organization (WHO) classification into mild (symptoms of upper respiratory tract infection, fever, fatigue, myalgia, GI symptoms, dyspnea), moderate (pneumonia with no need for supplemental oxygen) and severe/ critical disease (pneumonia with either respiratory rate $>30$ breaths/min or severe respiratory distress or hypoxemia with oxygen saturation $\leq 93 \%$; the presence of ARDS, acute kidney injury (AKI), or coagulation disorder) $[8,9]$. AKI was determined as reported by the author of original publication. Disease outcome was classified by whether the patient was discharged, still hospitalized or died at the time of case publication. Continuous variables were compared with the Wilcoxon rank-sum test or Kruskal-Wallis test and categorical variables using the chi-squared test or Fisher's exact test as applicable. We considered any missing variables as missing at random and analyzed only available data. All analyses were done using STATA (Version 14, StataCorp, College Station, Texas). The significance value was set at $\mathrm{P} \leq 0.05$.

\section{RESULTS}

Five hundred and sixty citations were identified through the database search and additional sources. Following the removal of duplicates, 288 unique citations were identified, and these were reviewed. Of the 288 citations, 69 full-text articles were assessed for eligibility. 57 articles fulfilled all the inclusion and exclusion criteria and were included in the review. Among these, 44 articles that included 149 COVID-19 cases in renal transplant recipients reported individual data and were selected for further univariate analysis by disease outcome and severity. 13 articles reported aggregate data on 561 renal transplant patients alone excluding other solid organ transplants and were tabulated in separate evidence table. The article selection process is outlined through PRISMA diagram [10] (Figure 1). Appraisal of study quality as well as assessment of risk of bias was conducted based on the NIH quality assessment tool. The studies were assessed for different criteria including study objective clearly stated, case definition, study population, subjects comparable, intervention clearly described, outcome measures clearly and consistently defined, length of follow up, result well-described or not etc. Majority of the studies included in this review were graded between good to fair with little or least risk of bias.

\section{OVERALL PATIENT CHARACTERISTICS}

44 articles reporting individual case data and 13 articles reporting aggregate data on renal transplant recipients were included in the review. There were articles with missing data for some of the variables. The missing data was considered to be missing at random and only the available data was analyzed and no imputation or statististical modeling was applied to account for missing data [11]. All the 44 articles included in the review except one were either case reports or case series reporting on 149 cases of COVID-19 in renal transplant recipients. The remaining non-case report article was a review article but was included due to the inclusion of a unique case report in the review. Baseline demographics, patient's presentation, results of radiological and immunological markers and COVID-19 severity of these 149 cases are summarized in Table 1. As demonstrated in Table 1, articles were globally representative with 16 articles originating from Asia [(China 12), Thailand (1), Iran (2), Korea (1)], 20 from Europe [Italy (8), Spain (2), France (2), Turkey (3), UK (2), Portugal (1), Poland (1), and Netherlands (1)], and 8 from America [North America (7), and South America (1)]. The average (range) age of patients included was 53 years (21-80 years) with the majority of the cases $(79 \%)$ being younger than 65 years of age. The majority of patients were men (75\%), with $81 \%$ of cases having at least one comorbidity with hypertension (HTN) $(71 \%)$, diabetes mellitus $(30 \%)$, and heart disease $(21 \%)$ being the most commonly reported comorbidities. The recipients were between 0 to 31 years post-renal transplant with the majority being hospitalized within 7 days of symptom onset predominantly with fever $(87 \%)$, cough $(58 \%)$, shortness of breath (SOB) $(50 \%)$, gastrointestinal (GI) symptoms (22\%), and myalgia (17\%). Among those reported, the majority had typical radiological picture of COVID-19 with bilateral lung involvement, and $94 \%$ had increased inflammatory markers including 
C-reactive protein (CRP), lactate dehydrogenase (LDH), ferritin, and d-dimer. Acute kidney allograft injury (AKI) developed in $71(49 \%)$ of the cases, $30(20 \%)$ developed adult respiratory distress syndrome (ARDS) while 69\% required supplemental oxygen therapy. When classified according to the WHO classification, $113(76 \%)$ cases had severe disease mainly due to development of hypoxia, AKI and/or ARDS. Main causes of disease severity are included in Table 1.

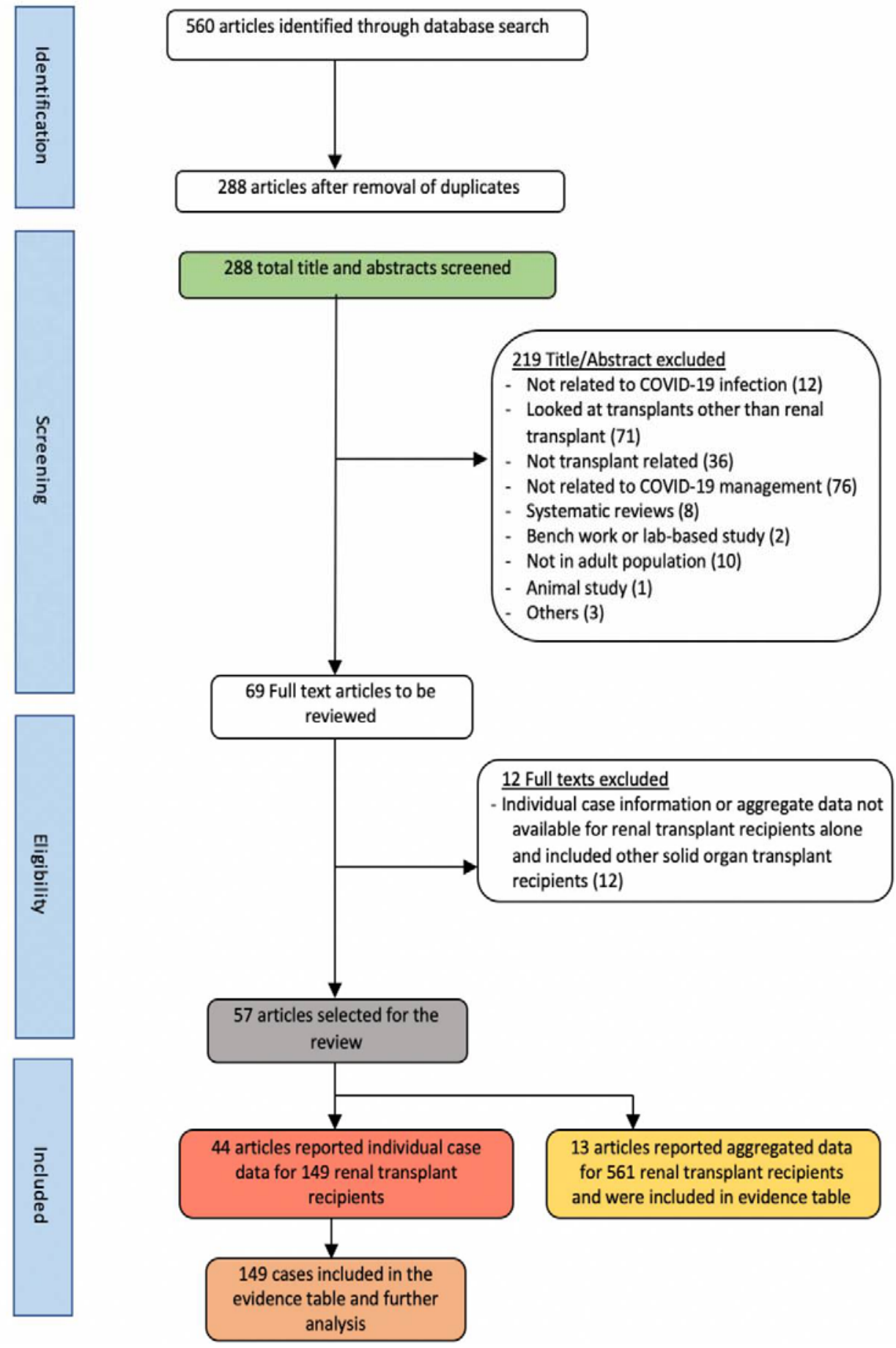

Figure 1. PRISMA flowchart summarizing the article selection process.

Table 2 summarizes the management and the clinical outcomes of the 144 patients. A combination of corticosteroids, calcineurin inhibitor (CNI) (especially tacrolimus) and antimetabolite (namely mycophenolate mofetil (MMF) and mycophenolic acid (MPA)) was the predominant maintenance immunosuppression. Of the 149 cases, 137 cases had reduction in their baseline immunosuppression. Only 
12 cases were managed without making changes to their baseline immunosuppressants with or without an increase in the dose of steroid therapy and none of these cases progressed to mechanical ventilation, and all fully recovered. COVID-19 specific treatment was provided in 128 of the 144 cases with mono or combination therapy including lopinavir/ritonavir (Lop/r), hydroxychloroquine (HCQ), oseltamivir, mizoribine (Miz), umifenovir, and darunavir/cobicistat (Dar/c). Of those articles who reported, 19 cases received monoclonal antibody tocilizumab and only 3 cases received remdesivir. Concomitant antibiotics was administered in $93(80 \%)$ of cases and $28(34 \%)$ received azithromycin. Among the 144 cases with reported outcome, death occurred in $34(23.6 \%)$ cases while $36(25 \%)$ patients remained hospitalized and 74 (51\%) patients were discharged from the hospital. Although mortality rate varied by month of publication but when time period stratified by early period (March to May) and late period (June-July) the mortality rate was found to be similar between the two time periods and similar to overall mortality rate. The average duration from onset of symptom to outcome was 23.6 days. There were two cases of non-biopsy proven graft rejection and 1 case of hemophagocytic disease and thromboembolic disease each $[12,13]$.

Table 3 summarizes the findings from studies reporting aggregate data for management of COVID-19 in 561 renal transplant recipients. Among the 13 articles, 2 originated from Asia [Iran (2)], 6 from Europe [Spain (4), Italy (1) and Turkey (1)] and 5 from USA [North America (4) and South America (1)]. As demonstrated in the table, the average age ranged between 45 to 75 years with majority of patients being male. Hypertension was the commonly reported comorbidity followed by diabetes and heart disease. The median time since transplant ranged between 0.09 years to 10.7 years. Predominant symptoms included fever, shortness of breath and diarrhea. When reported AKI was present in 23\% to $74 \%$ of the patients. For majority of the patients' immune markers including CRP, LDH and d-Dimer was elevated. CNI, MMF and steroid was the most common baseline immunosuppressants used which in most of the cases were either reduced or withdrawn followed by maintenance therapy with steroid. Covid-19 specific treatment was used in majority of cases with mostly HCQ being used followed by Lop/r and antibiotics. Tocilizumab was reported in $7 \%$ to $74 \%$ of patients. The mortality rate ranged between $6 \%$ to $50 \%$. All the studies were published between May and July. The average mortality rate was found to be $26 \%$ which was similar to the mortality rate of $23.6 \%$ reported by studies with individual case data.

\section{CHARACTERISTICS AND FACTORS AFFECTING DISEASE SEVERITY}

\section{Demographics, clinical, laboratory, and immunological factors}

Table 4 summarizes factors associated with disease severity. As demonstrated in Table 4, the majority of patients were hospitalized within 7 days from symptom onset with no significant difference between disease severity groups. There was no significant difference between patients with mild/ moderate disease and those with severe disease in terms of age $(\mathrm{P}=0.15)$, gender $(\mathrm{P}=0.66)$, comorbidities $(\mathrm{P}>0.05)$ and baseline immunosuppression with the exception of corticosteroid which was utilized more in patients with severe disease. When reported, ACEi/ARB was more commonly used in patients with severe disease $(34 \%$ vs $6 \%, \mathrm{P}=0.03)$. More cases with severe disease had radiological evidence of bilateral lung involvement ( $84 \%$ vs $36 \%$ ) with only $8 \%$ of severe disease cases compared to $46 \%$ of mild/moderate disease had no lung involvement $(\mathrm{P}=0.00)$. Although the proportion of patients with lymphopenia was comparable between the groups, patients with severe disease had higher CRP $(\mathrm{P}=0.005)$, higher LDH $(\mathrm{P}=0.0004)$, higher Ferritin $(\mathrm{P}=0.05)$, higher d-Dimer $(0.008)$ and higher serum creatinine at presentation $(\mathrm{P}<0.001)$. Overall, 34\% of cases required ICU support. Patients with severe disease were more likely to require ICU care (Table 4 ).

Reduction or withdrawal of baseline immunosuppressant was more commonly undertaken in patients with severe disease as compared to mild/moderate disease $(96 \%$ vs $78 \%, \mathrm{P}=0.001)$ mainly by CNI reduction or elimination $(73 \%$ vs $44 \%, \mathrm{P}=0.002)$ and an increase in corticosteroid use $(69 \%$ vs $31 \%, P=0.00)$. There was no significant difference between groups in the antimetabolite dose reduction. Significant differences were also observed among the groups in terms of antiviral treatment. Among the severe versus mild/moderate disease cases, $47 \%$ vs. $14 \%, 6 \%$ vs $20 \%$ and $76 \%$ vs. $43 \%$ received Lop $/ \mathrm{r}(\mathrm{p}=0.001)$, oseltamivir $(p=0.02)$ and HCQ $(p=0.00)$ respectively. The immunomodulatory agent tocilizumab was used in 19 cases all of which were with severe disease. Of those who received tocilizumab, 10 required intubation and 6 required ICU management and 7 died. Antibacterial therapy was used in $87 \%$ of severe cases as compared to $60 \%$ of $\mathrm{mild} / \mathrm{moderate}$ disease $(\mathrm{p}=0.001)$ with no significant difference in azithromycin use between the groups $(\mathrm{P}=0.78)$. 


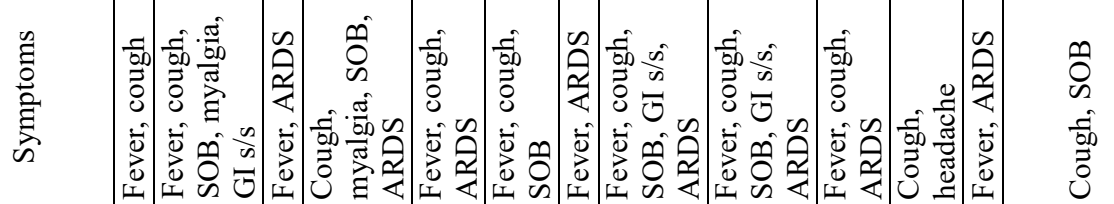

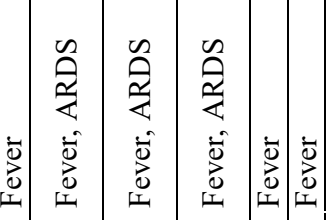

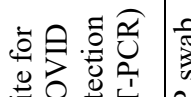

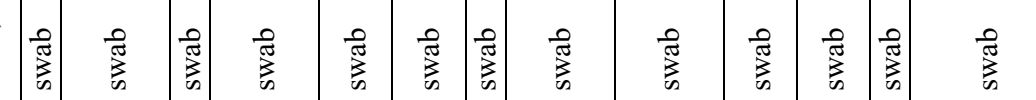

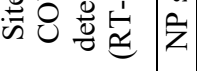

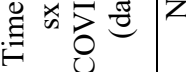

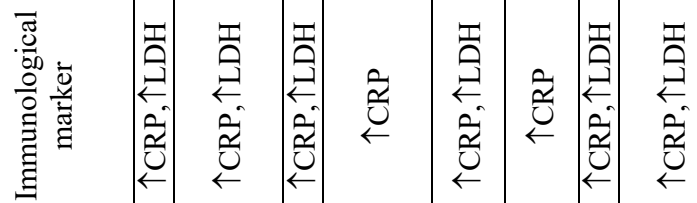

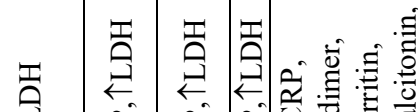

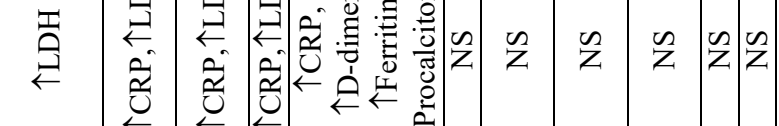

事范

总至焉

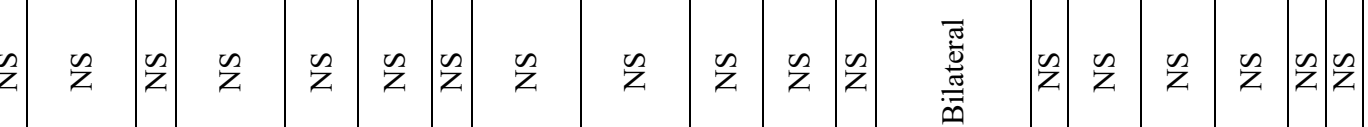
$\bar{v}$

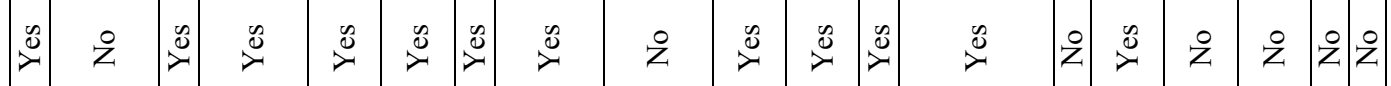

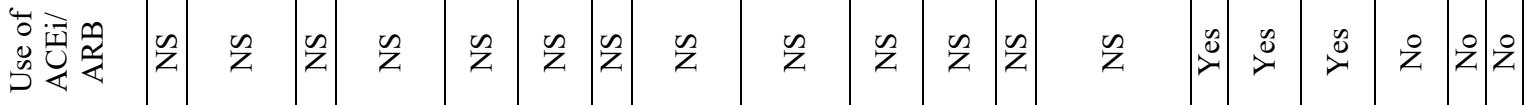

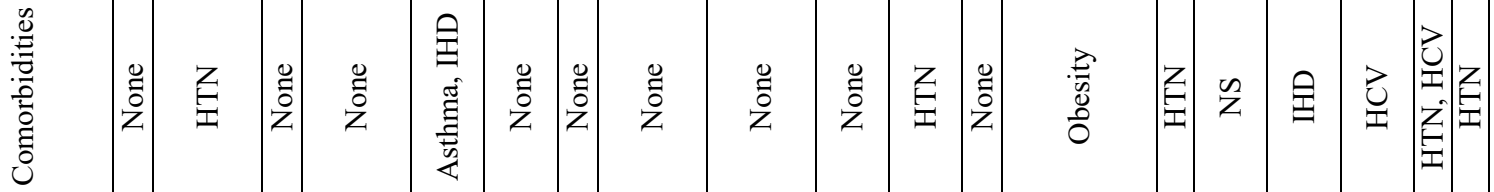

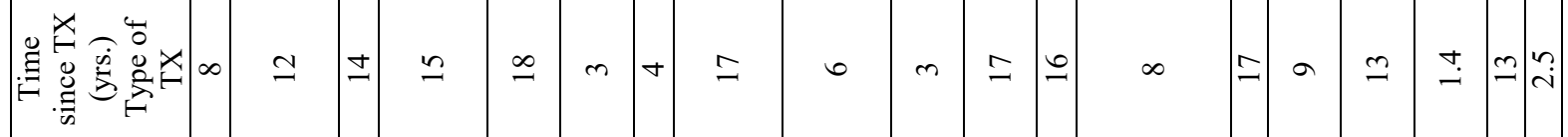

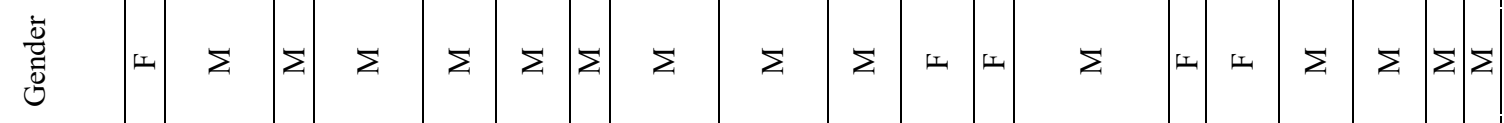

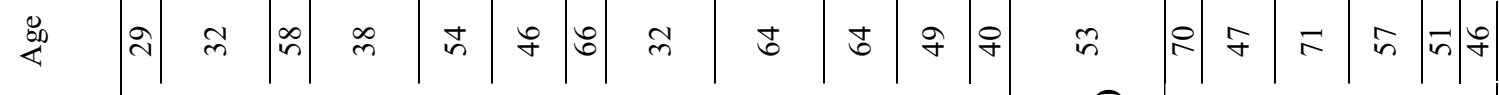

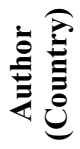




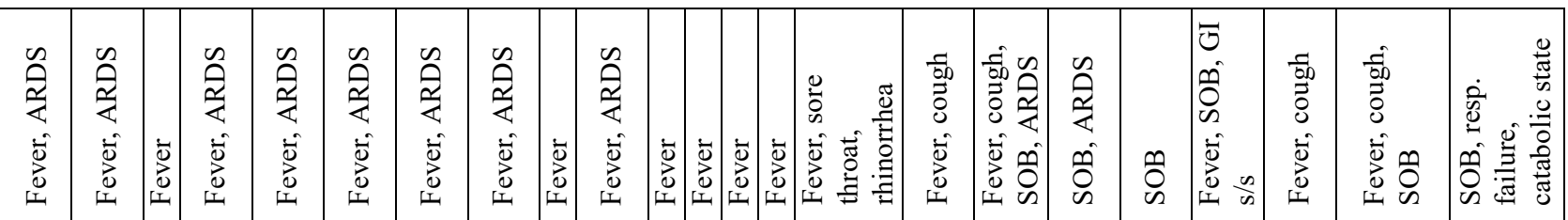

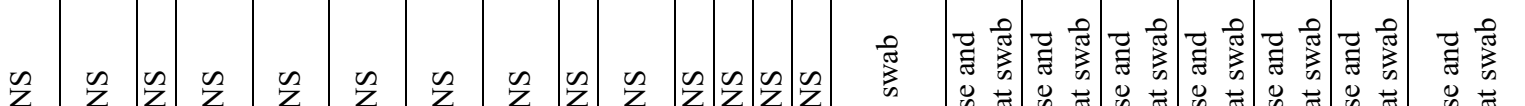

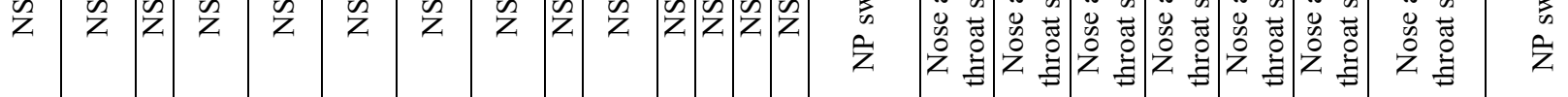

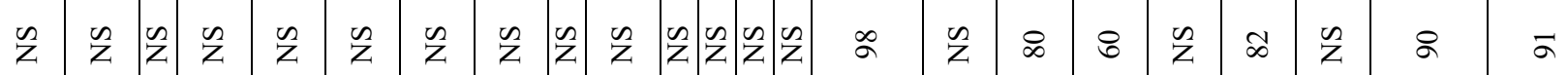

$\tilde{Z}$ Z

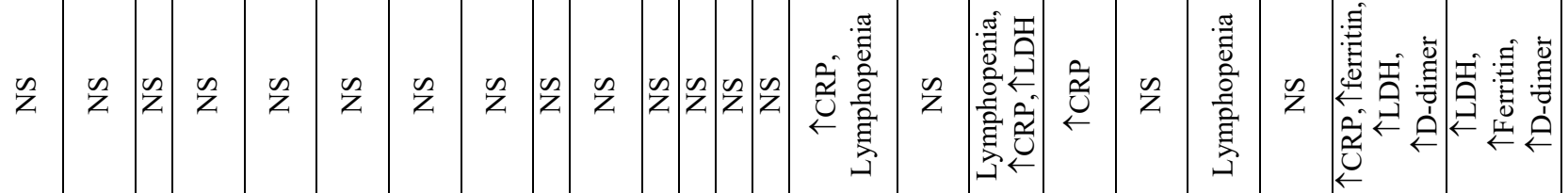

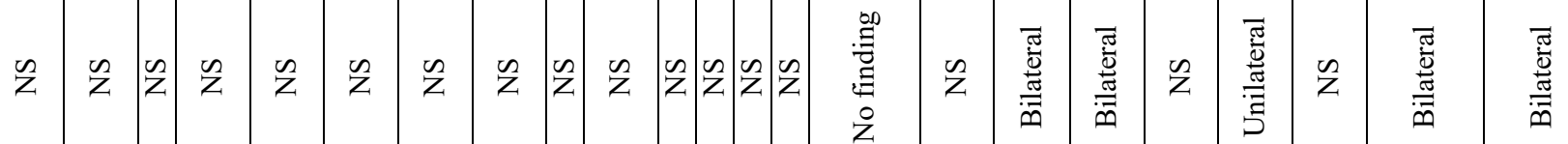

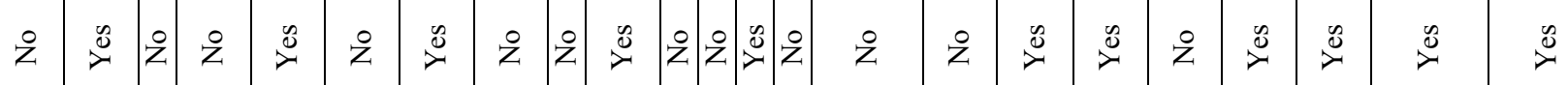

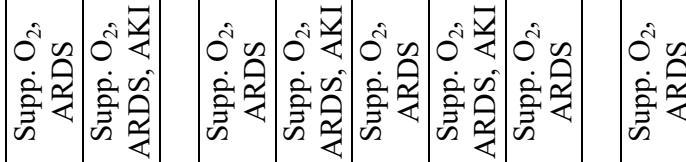

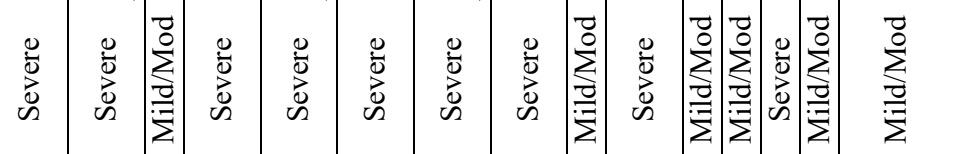

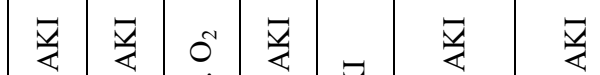

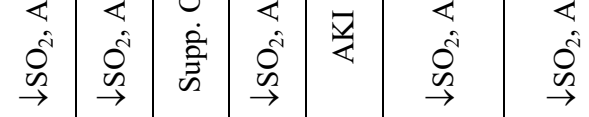

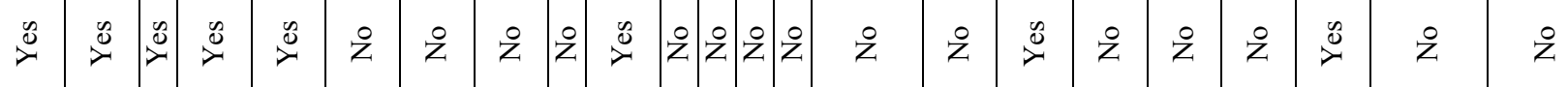

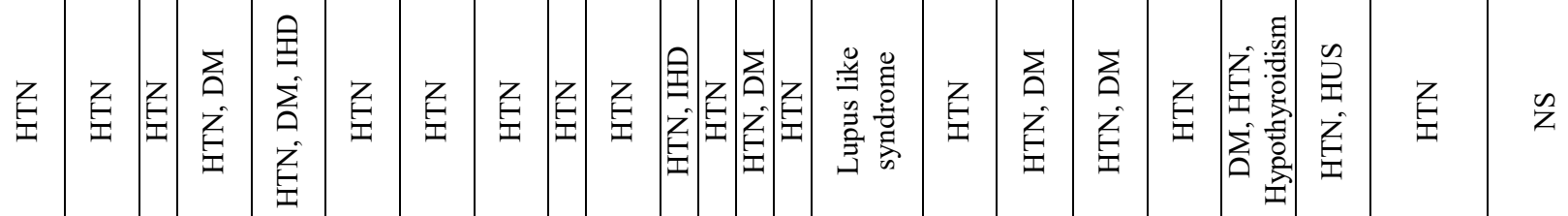

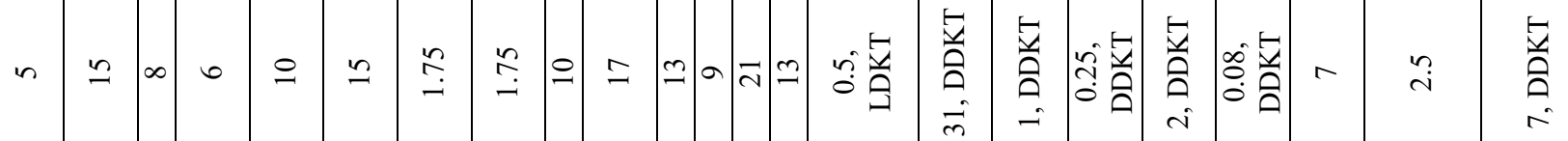

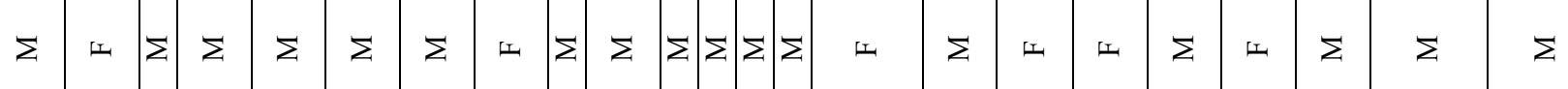

in 암

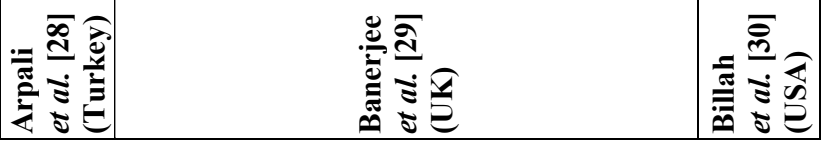









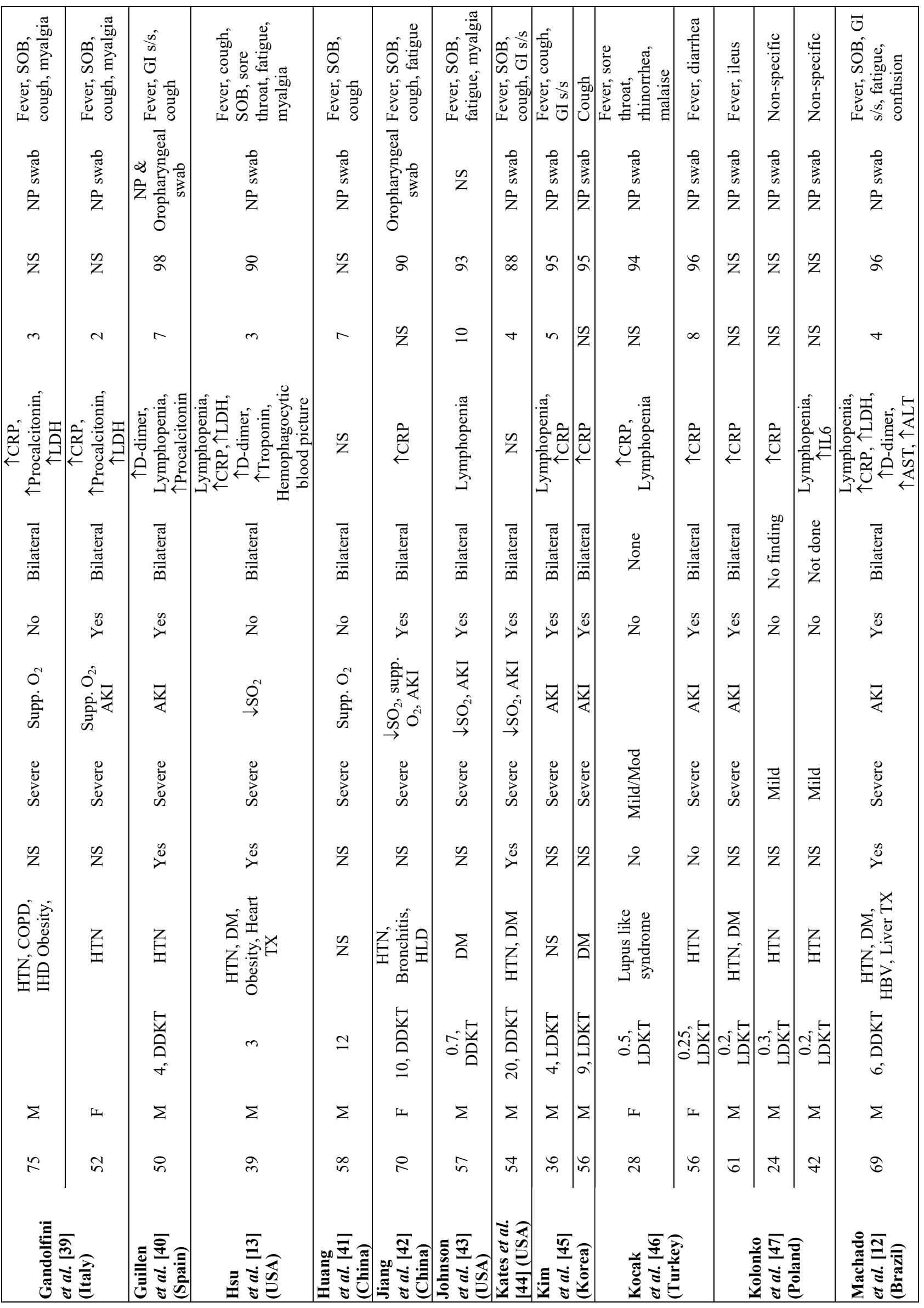




\begin{tabular}{|c|c|c|c|c|c|c|c|c|c|c|c|c|}
\hline 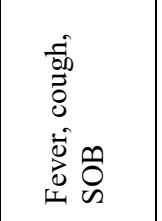 & 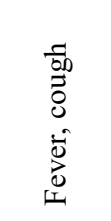 & $\begin{array}{l}m \\
\text { o }\end{array}$ & 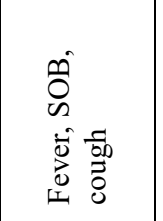 & 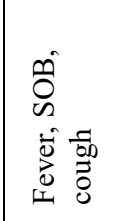 & $\begin{array}{l}\bar{c} \\
\overline{0} \\
\overline{0} \\
\dot{0} \\
\overrightarrow{0} \\
\end{array}$ & 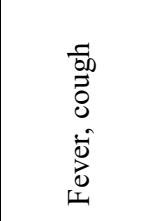 & 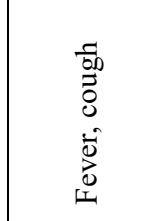 & 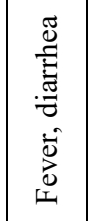 & 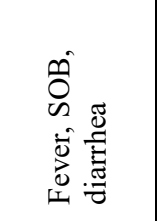 & 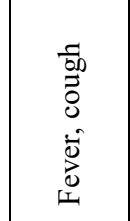 & 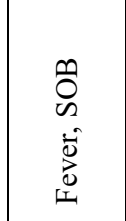 & 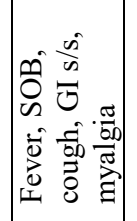 \\
\hline 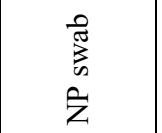 & 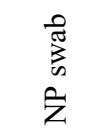 & 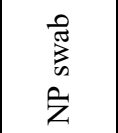 & 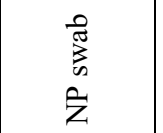 & 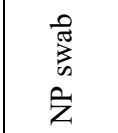 & 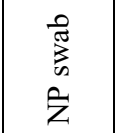 & 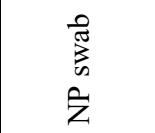 & 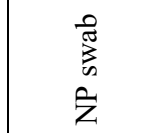 & 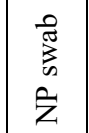 & 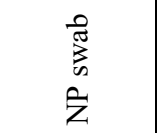 & 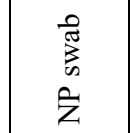 & 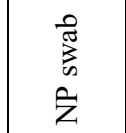 & 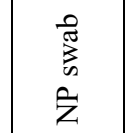 \\
\hline$\stackrel{\infty}{\circ}$ & $\tilde{z}$ & a & $\tilde{Z}$ & $\stackrel{\infty}{\sigma}$ & 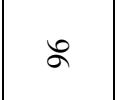 & $\stackrel{\circ}{\hat{v}}$ & $\stackrel{\circ}{\grave{v}}$ & $\stackrel{\circ}{\grave{v}}$ & $\stackrel{\grave{\hat{v}}}{\grave{v}}$ & $\stackrel{\circ}{\grave{v}}$ & $\stackrel{\circ}{\grave{v}}$ & જे \\
\hline$\tilde{z}$ & $\infty$ & $\tilde{z}$ & 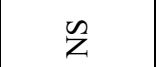 & $\tilde{z}$ & 6 & $\tilde{z}$ & 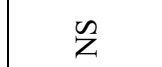 & $\tilde{z}$ & $\tilde{z}$ & $\tilde{z}$ & $\tilde{z}$ & 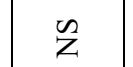 \\
\hline 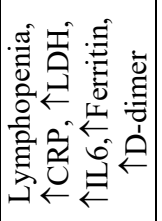 & 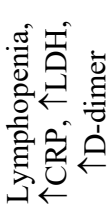 & 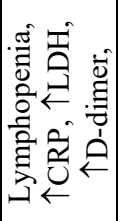 & 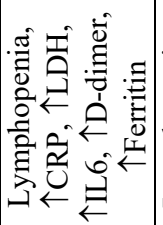 & 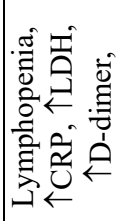 & 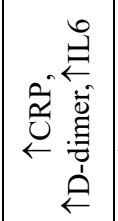 & 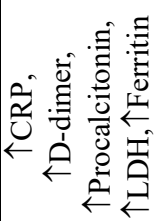 & 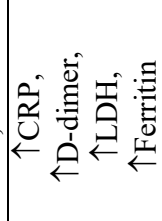 & 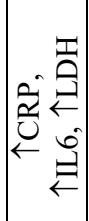 & 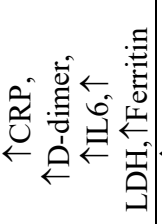 & 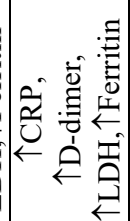 & 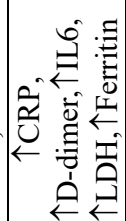 & 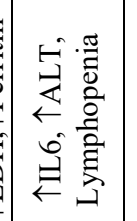 \\
\hline 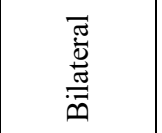 & 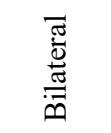 & 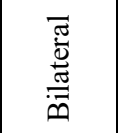 & 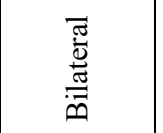 & 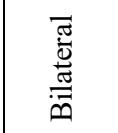 & 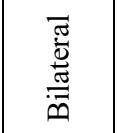 & $\tilde{Z}$ & $\tilde{Z}$ & $\tilde{z}$ & $\tilde{Z}$ & $\tilde{z}$ & $\tilde{z}$ & 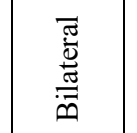 \\
\hline$\stackrel{8}{ }$ & $\stackrel{2}{z}$ & $\check{z}$ & $\stackrel{\infty}{0}$ & 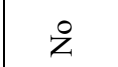 & $\stackrel{z}{z}$ & $\stackrel{\infty}{\check{\nu}}$ & $\stackrel{\infty}{\infty}$ & $\tilde{z}$ & $\tilde{z}$ & $\tilde{z}$ & $\tilde{z}$ & $\stackrel{\infty}{\infty}$ \\
\hline 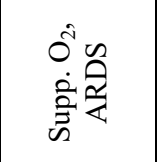 & 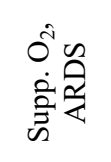 & $\begin{array}{l}0 \\
\dot{0} \\
\dot{\vec{\Xi}}\end{array}$ & 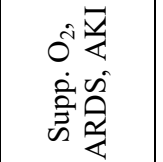 & 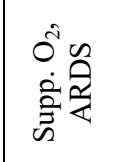 & & 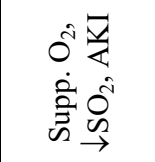 & 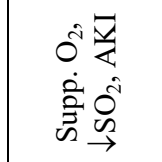 & 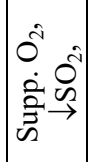 & $\begin{array}{l}\hat{0} \\
\dot{2} \\
\dot{\vec{g}} \\
\hat{0}\end{array}$ & 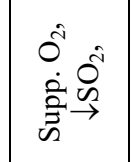 & 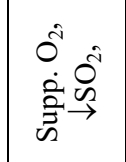 & $\bar{z}$ \\
\hline 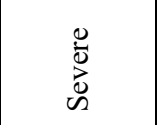 & 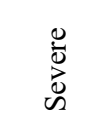 & $\begin{array}{l}0 \\
\text { Dे } \\
\text { D. }\end{array}$ & $\begin{array}{l}0 \\
\text { Dे } \\
\text { D. }\end{array}$ & $\begin{array}{l}0 \\
\text { Dे } \\
\text { D. }\end{array}$ & 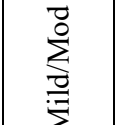 & 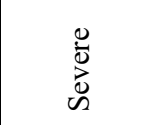 & 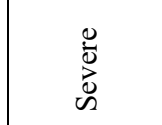 & 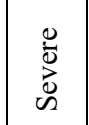 & $\begin{array}{l}\text { D. } \\
\text { Dे } \\
\tilde{n}\end{array}$ & $\begin{array}{l}0 \\
\text { Dे } \\
\text { D. }\end{array}$ & $\begin{array}{l}\text { गे } \\
\bar{D} \\
\tilde{n}\end{array}$ & 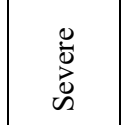 \\
\hline$\stackrel{z}{z}$ & $\stackrel{\gtrless}{z}$ & $\stackrel{z}{z}$ & $\stackrel{2}{z}$ & $\stackrel{2}{z}$ & $\stackrel{z}{z}$ & $\tilde{z}$ & $\tilde{z}$ & $\stackrel{z}{z}$ & $\tilde{z}$ & ż & $\stackrel{z}{z}$ & $\stackrel{z}{z}$ \\
\hline$\underset{I}{Z}$ & 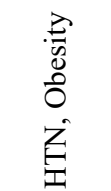 & 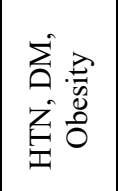 & 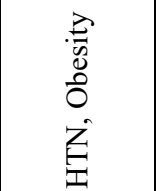 & $\begin{array}{l}\text { 悹 } \\
\text { 昜 }\end{array}$ & 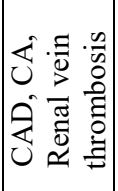 & 忘 & $\begin{array}{l}\sum_{i}^{0} \\
\text { 至已 }\end{array}$ & 己 & 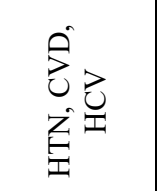 & $\begin{array}{l}3 \\
0 \\
0 \\
0\end{array}$ & $\begin{array}{l}2 \\
己 \\
z \\
z \\
z\end{array}$ & $\tilde{z}$ \\
\hline $\begin{array}{l}\vec{y} \\
\vec{G} \\
=\end{array}$ & $\begin{array}{l}\bar{a} \\
\overrightarrow{0} \\
\dot{+}\end{array}$ & ণ̋: & 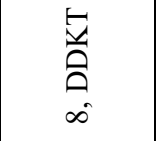 & $\begin{array}{l}5 \\
\stackrel{5}{0} \\
\end{array}$ & $N$ & $\begin{array}{l}0 \\
\dot{I}\end{array}$ & $\underset{0}{\tilde{O}}$ & $\begin{array}{l}\text { r. } \\
\dot{r}\end{array}$ & 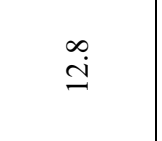 & $\stackrel{\circ}{\stackrel{\circ}{I}}$ & $\begin{array}{l}0 \\
\infty \\
\infty\end{array}$ & $\nabla$ \\
\hline$\Sigma$ & 山 & $\Sigma$ & $\Sigma$ & 山 & $\Sigma$ & $\Sigma$ & $\Sigma$ & $\Sigma$ & $\Sigma$ & $\Sigma$ & $\Sigma$ & $\Sigma$ \\
\hline$\vec{n}$ & 6 & $\approx$ & $\mathbb{N}$ & $F$ & $\stackrel{\infty}{n}$ & $F$ & 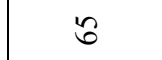 & in & $\widetilde{\sigma}$ & gे & $\widehat{\sigma}$ & 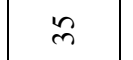 \\
\hline & & 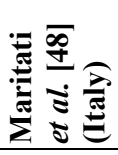 & & & 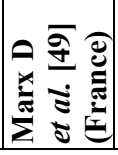 & \multicolumn{6}{|c|}{ 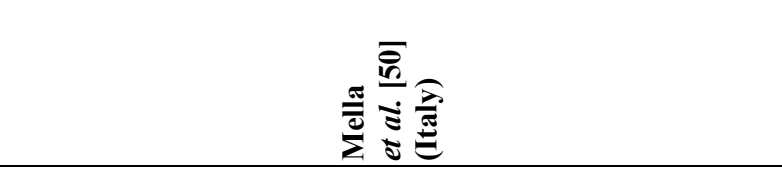 } & 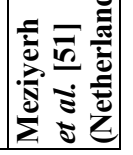 \\
\hline
\end{tabular}




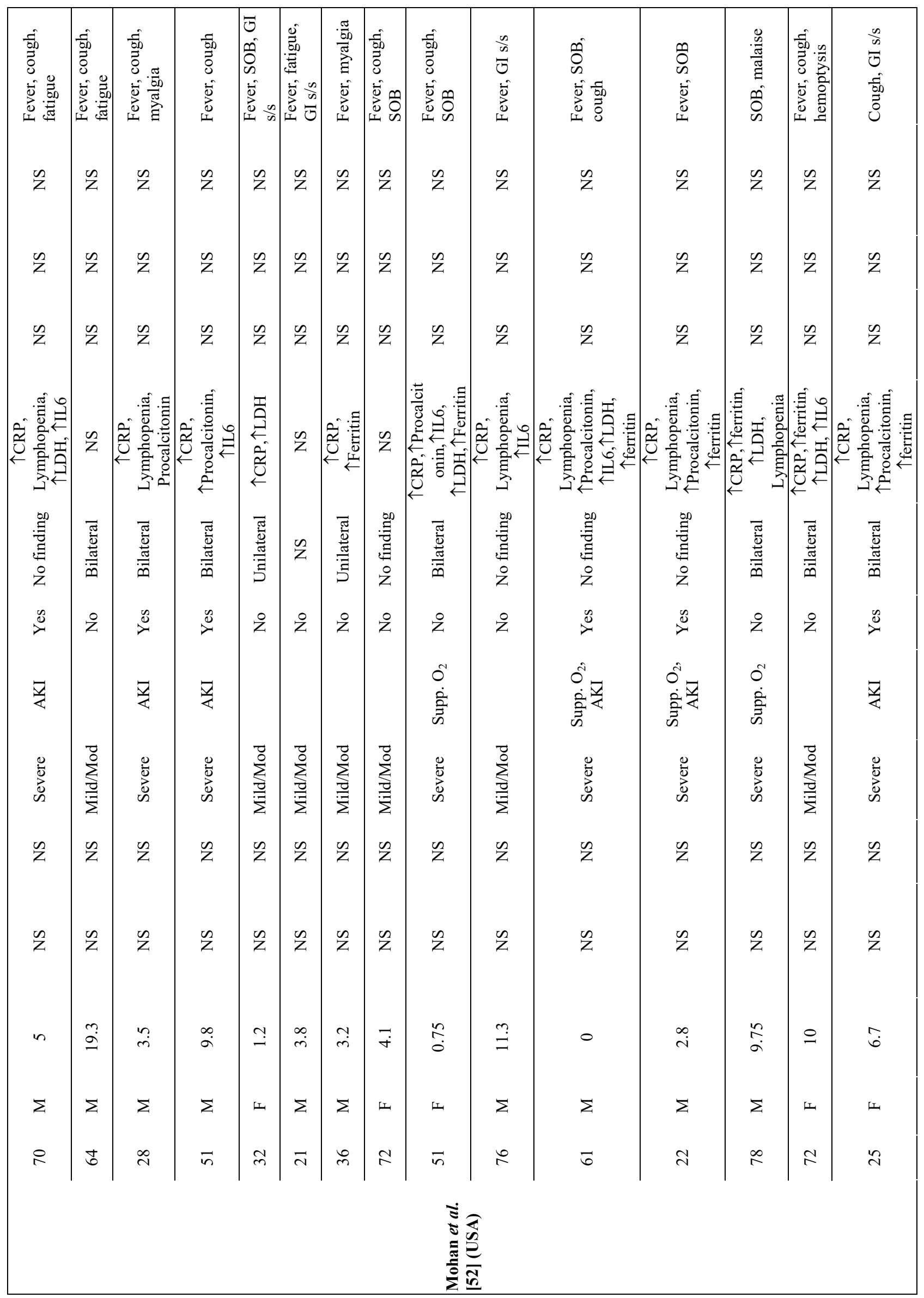




\begin{tabular}{|c|c|c|c|c|c|c|c|c|c|c|c|c|c|c|c|c|c|c|c|}
\hline 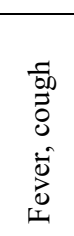 & 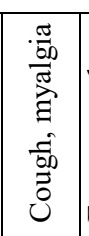 & 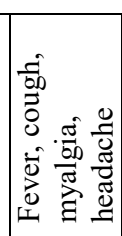 & 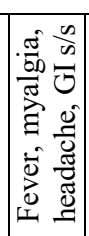 & 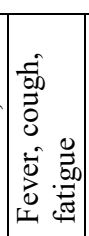 & 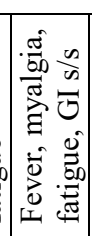 & 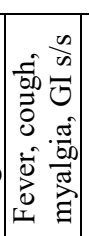 & 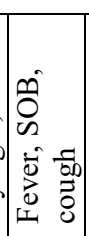 & 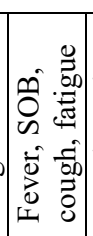 & 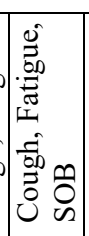 & $\begin{array}{l}\text { ô } \\
0 \\
\text { की } \\
\text { है } \\
0\end{array}$ & 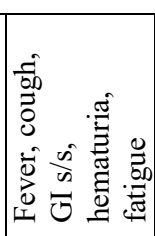 & 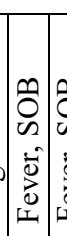 & 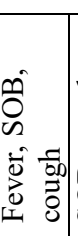 & 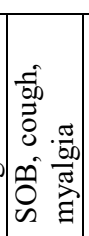 & 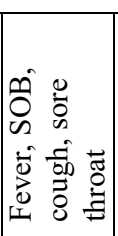 & $\left|\begin{array}{c}n \\
n \\
0 \\
0 \\
\dot{0} \\
\overrightarrow{0} \\
0 \\
1\end{array}\right|$ & 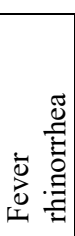 & 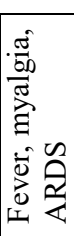 & 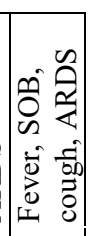 \\
\hline 莡 & 雚 & 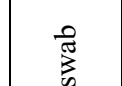 & 雚 & 㻤 & 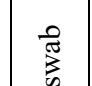 & 䓠 & 嚆 & 㻤 & 莺 & 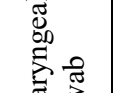 & 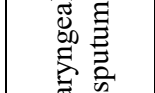 & 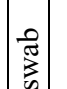 & $\frac{\pi}{\pi}$ & 钻 & 繁 & 䓠 & 傿 & 繁 & 8 \\
\hline 言 & 㝋 & 之े & $\hat{z}$ & $\hat{z}$ & $\hat{z}$ & $\hat{z}$ & $\hat{z}$ & $\hat{z}$ & $\hat{z}$ & 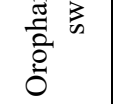 & 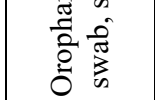 & $\left|\begin{array}{l}\infty \\
\mathbf{z}\end{array}\right|$ & $\hat{z}$ & $\hat{z}$ & $\hat{z}$ & $\hat{z}$ & $\hat{z}^{\infty}$ & $\hat{z}$ & $n$ \\
\hline$\tilde{z}$ & $\tilde{z}$ & $\tilde{z}$ & $\tilde{z}$ & $\tilde{z}$ & $\tilde{z}$ & $\tilde{z}$ & $\tilde{z}$ & $\tilde{Z}$ & $\tilde{z}$ & $\stackrel{\infty}{\sim}$ & $\tilde{z}$ & $\infty$ & 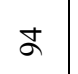 & 8 & $\hat{a}$ & 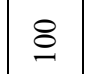 & 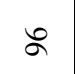 & 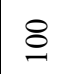 & ๙ \\
\hline 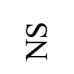 & $\tilde{z}$ & $\tilde{z}$ & Z & Z & $\tilde{z}$ & $\tilde{z}$ & $\tilde{z}$ & $\tilde{z}$ & $\tilde{z}$ & $r$ & $\sim$ & 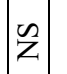 & $\tilde{z}$ & $\tilde{z}$ & z & $\tilde{z}$ & $\tilde{z}$ & $\tilde{Z}$ & Z \\
\hline $\begin{array}{l}\mathscr{0} \\
\check{Z}\end{array}$ & 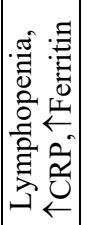 & $\stackrel{\approx}{\longleftarrow}$ & Z̃ & 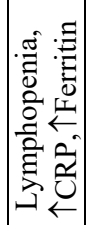 & 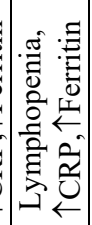 & 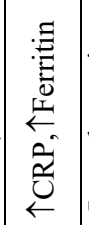 & 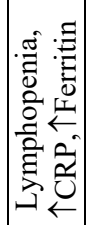 & 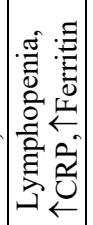 & 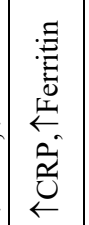 & 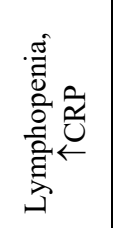 & $\tilde{z}$ & $\tilde{z}$ & $\stackrel{\circ}{\rightleftarrows}$ & $\tilde{z}$ & $\stackrel{\circ}{\rightleftarrows}$ & $\tilde{z}$ & $\tilde{Z}$ & $\tilde{Z}$ & $\tilde{z}$ \\
\hline 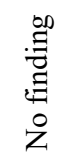 & 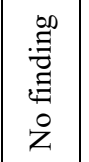 & $\begin{array}{l}\bar{\pi} \\
\frac{\pi}{0} \\
\frac{\pi}{3} \\
5\end{array}$ & 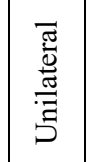 & 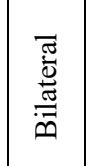 & 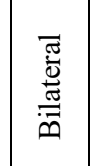 & 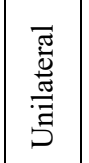 & 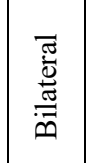 & 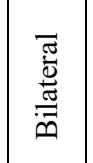 & 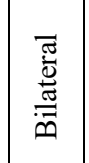 & $\begin{array}{l}\bar{\pi} \\
\frac{\pi}{\overline{0}} \\
\frac{\pi}{\bar{m}}\end{array}$ & 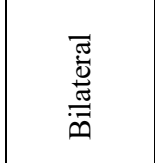 & 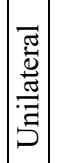 & $\begin{array}{l}\bar{\pi} \\
\frac{\pi}{0} \\
\frac{\pi}{\pi} \\
5\end{array}$ & 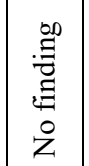 & $\begin{array}{l}\bar{\pi} \\
\frac{\pi}{\tilde{m}} \\
\frac{\pi}{\bar{n}}\end{array}$ & 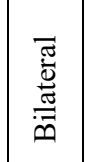 & 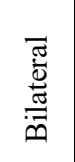 & 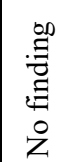 & 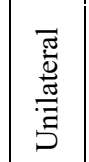 \\
\hline ż & $\stackrel{8}{z}$ & \& & z & $\stackrel{0}{\nu}$ & 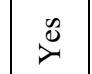 & $\stackrel{\infty}{\infty}$ & $\stackrel{\infty}{\infty}$ & $\stackrel{8}{z}$ & $\stackrel{\infty}{\nu}$ & $\stackrel{\infty}{\infty}$ & $\stackrel{\infty}{\infty}$ & 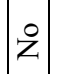 & z & z & z & z & $\ddot{z}$ & $\stackrel{2}{z}$ & $\sum_{\nu}^{\infty}$ \\
\hline & & & & 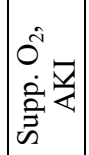 & 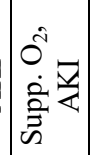 & 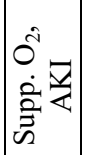 & 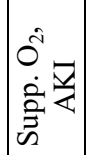 & $\begin{array}{l}0 \\
0 \\
\dot{2} \\
\stackrel{\vec{B}}{n}\end{array}$ & 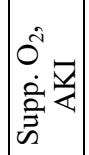 & 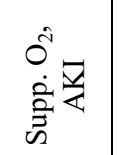 & $\frac{\vec{z}}{4}$ & 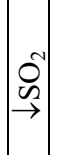 & 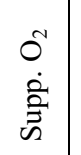 & $\begin{array}{l}\delta^{2} \\
\stackrel{\mathscr{C}}{\rightarrow}\end{array}$ & $\begin{array}{l}0 \\
0 \\
\dot{2} \\
\hat{\Xi}\end{array}$ & $\begin{array}{l}0 \\
\dot{0} \\
\hat{\vec{\Xi}} \\
\text { के }\end{array}$ & $\begin{array}{l}0 \\
\dot{0} \\
\hat{\mathbf{n}} \\
\text { की }\end{array}$ & 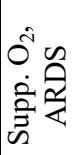 & $\underset{c}{0}$ \\
\hline$\sum_{\substack{z \\
z}}^{z}$ & $\sum_{\substack{0\\
}}^{\overrightarrow{0}}$ & 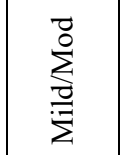 & 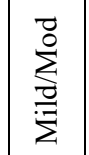 & 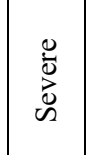 & 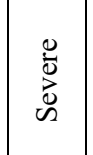 & $\begin{array}{l}\text { Dे } \\
\vec{D} \\
\ddot{D}\end{array}$ & $\begin{array}{l}0 \\
\stackrel{D}{0} \\
\ddot{D}\end{array}$ & $\begin{array}{l}\text { D. } \\
\dot{D} \\
\ddot{D}\end{array}$ & 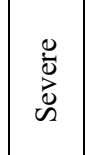 & $\begin{array}{l}\stackrel{D}{D} \\
\vec{D} \\
\mathscr{D}\end{array}$ & 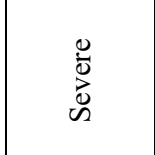 & 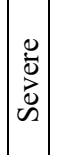 & $\begin{array}{l}0 \\
\stackrel{\bar{D}}{0} \\
\stackrel{0}{n}\end{array}$ & $\begin{array}{l}0 \\
\grave{D} \\
\vdots \\
\end{array}$ & 离 & 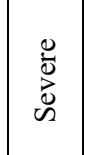 & $\begin{array}{l}0 \\
\stackrel{0}{0} \\
\dot{0} \\
\end{array}$ & 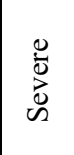 & $\bar{\nabla}$ \\
\hline$\tilde{z}$ & $\bar{z}$ & $\tilde{z}$ & $\tilde{z}$ & $\tilde{z}$ & $\bar{z}$ & $\bar{z}$ & $\tilde{z}$ & z & $\tilde{z}$ & $\tilde{z}$ & $\stackrel{\infty}{\varnothing}$ & $\stackrel{\gtrless}{2}$ & $\stackrel{0}{\infty}$ & $\stackrel{2}{2}$ & $\stackrel{2}{2}$ & $\stackrel{\gtrless}{z}$ & $\stackrel{\infty}{\varnothing}$ & $\stackrel{\infty}{\infty}$ & z \\
\hline 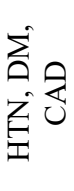 & $\begin{array}{l}\sum \\
\dot{\partial} \\
Z \\
\text { 至 }\end{array}$ & 忘 & 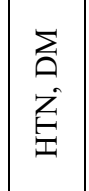 & \begin{tabular}{l|} 
主 \\
Ż \\
至
\end{tabular} & 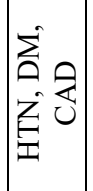 & $\begin{array}{l}\sum \\
\text { 至 } \\
z \\
\text { 至 }\end{array}$ & \begin{tabular}{l|} 
主 \\
Ż \\
至
\end{tabular} & $\begin{array}{l}\text { 感 } \\
z \\
\text { 至 }\end{array}$ & \begin{tabular}{l|} 
主 \\
Ż \\
至
\end{tabular} & 䚾 & 壱 & $\mid \begin{array}{l}\mathbb{u} \\
0 \\
z \\
\vdots \\
\vdots\end{array}$ & $\begin{array}{l}\sum_{2} \\
\text { 至 }\end{array}$ & 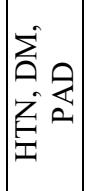 & 昇 & 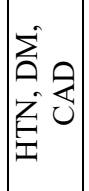 & 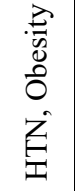 & 㽦 & 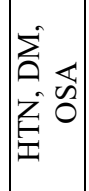 \\
\hline mi & 章 & $\begin{array}{l}\overrightarrow{4} \\
\vec{a} \\
a\end{array}$ & 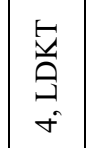 & 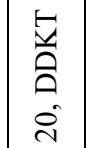 & 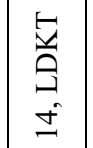 & $\begin{array}{l}\vec{y} \\
\dot{0} \\
m\end{array}$ & $=$ & $\begin{array}{l}\frac{5}{3} \\
a \\
a\end{array}$ & $\begin{array}{l}\overrightarrow{\mathrm{a}} \\
\overrightarrow{0} \\
\dot{m}\end{array}$ & 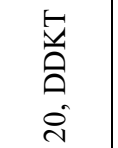 & 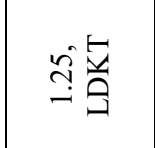 & $\begin{array}{l}m \\
\infty \\
\infty\end{array}$ & $\stackrel{\infty}{\rightarrow}$ & $\stackrel{\infty}{\infty}$ & 0 & $\overrightarrow{\dot{m}}$ & $\begin{array}{l}\infty \\
\dot{\Xi}\end{array}$ & 㐫 & $?$ \\
\hline$\Sigma$ & $\Sigma$ & L & II & $\Sigma$ & $\Sigma$ & $\Sigma$ & $\Sigma$ & L & I & 山 & $\Sigma$ & & $\Sigma$ & $\Sigma$ & 工 & $\Sigma$ & $\Sigma$ & $\Sigma$ & $z$ \\
\hline in & $\hat{n}$ & 8 & $\bar{m}$ & in & 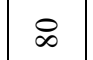 & if & $\ddot{0}$ & $\approx$ & in & $\beta$ & ते & 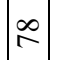 & $\approx$ & 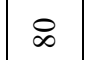 & $\nabla$ & $F$ & 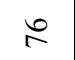 & ले & 6 \\
\hline \multicolumn{10}{|c|}{ 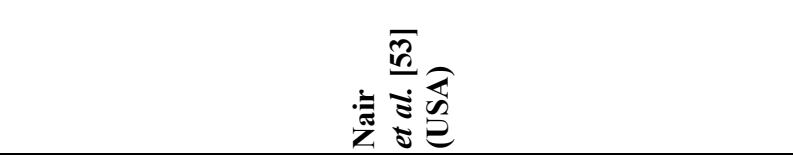 } & 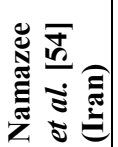 & 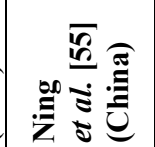 & \multicolumn{8}{|c|}{ 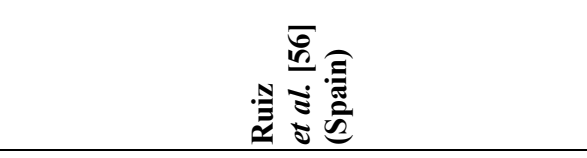 } \\
\hline
\end{tabular}




\begin{tabular}{|c|c|c|c|c|c|c|c|c|c|c|c|c|c|c|c|}
\hline $\begin{array}{l}\bar{c} \\
\text { b0 } \\
\overrightarrow{0} \\
\dot{0} \\
\ddot{0} \\
\vec{D}_{1}\end{array}$ & $\begin{array}{l}\overline{50} \\
\overrightarrow{0} \\
\overline{0}\end{array}$ & 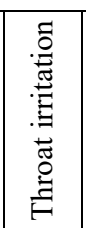 & 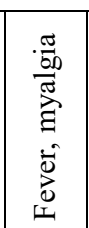 & 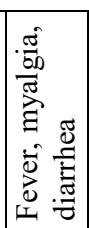 & 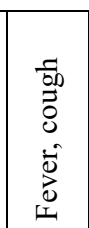 & 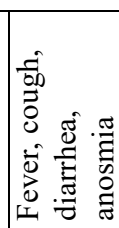 & 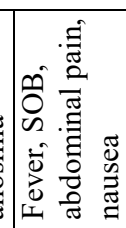 & 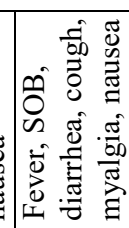 & 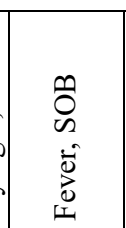 & 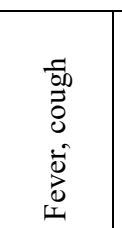 & 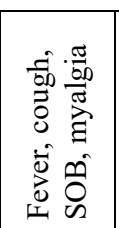 & 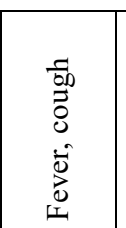 & 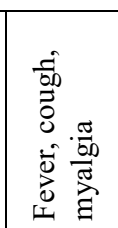 & 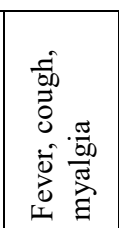 & 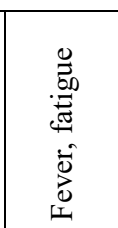 \\
\hline $\begin{array}{l}\frac{0}{\pi} \\
3 \\
0 \\
n\end{array}$ & 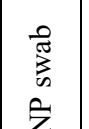 & 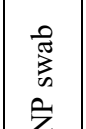 & $\begin{array}{l}0 \\
\text { 离 } \\
5\end{array}$ & 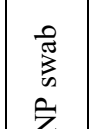 & 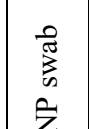 & 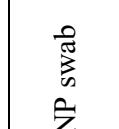 & 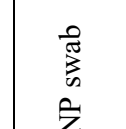 & 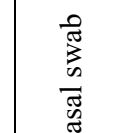 & 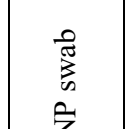 & 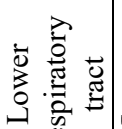 & 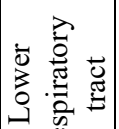 & 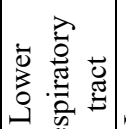 & 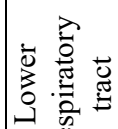 & 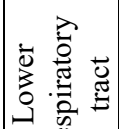 & 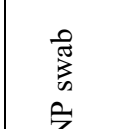 \\
\hline & $\bar{Z}$ & $\bar{Z}$ & & z & & $\bar{z}$ & $\bar{Z}$ & $\ddot{z}$ & $\bar{Z}$ & $\stackrel{0}{0}$ & - & b & $\underset{b}{\infty}$ & $\stackrel{0}{\infty}$ & $\overline{\mathbf{Z}}$ \\
\hline Z & aे & $\infty$ & & $\stackrel{\grave{\vartheta}}{\hat{v}}$ & & 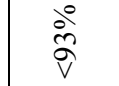 & 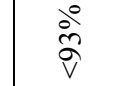 & a & $\tilde{z}^{n}$ & aे & $\approx$ & ๙े & $\stackrel{\infty}{\sigma}$ & $\hat{a}$ & $\tilde{z}$ \\
\hline$a$ & z & $\tilde{z}$ & $\simeq$ & \pm & r & $\infty$ & o & 2 & $r$ & z & $z$ & $\tilde{z}$ & $\sum z$ & Z & 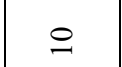 \\
\hline & 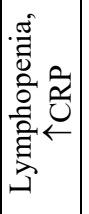 & 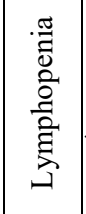 & 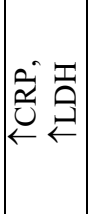 & 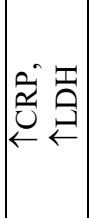 & 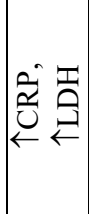 & 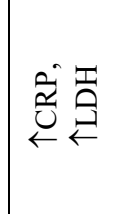 & 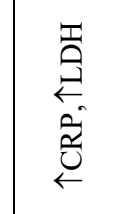 & 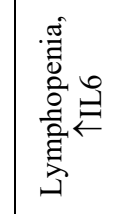 & 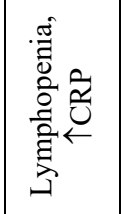 & 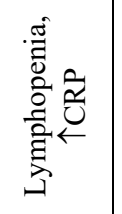 & 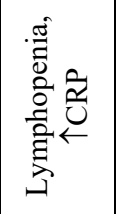 & 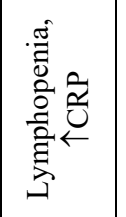 & 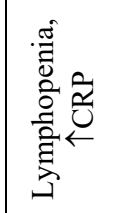 & 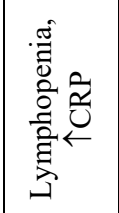 & 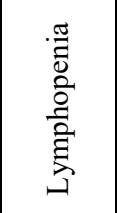 \\
\hline 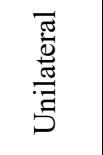 & 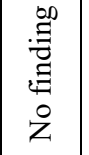 & 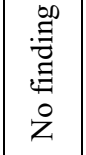 & 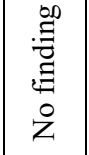 & 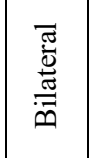 & 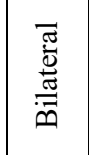 & 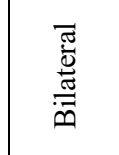 & 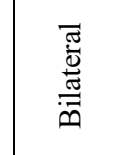 & 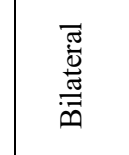 & 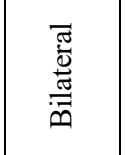 & 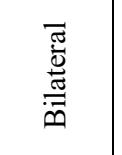 & 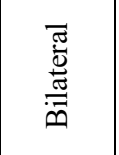 & 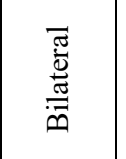 & 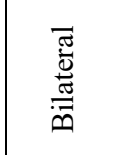 & 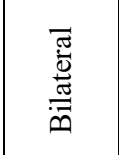 & 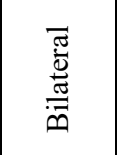 \\
\hline ż & 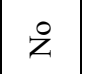 & 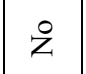 & $z$ & $\stackrel{\infty}{\nu}$ & $\stackrel{2}{z}$ & $\stackrel{\infty}{\infty}$ & $\stackrel{\varnothing}{\nu}$ & $\stackrel{\infty}{\nu}$ & $\stackrel{y}{\nu}$ & 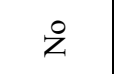 & $\stackrel{\infty}{\nu}$ & z & $\stackrel{\circ}{z}$ & 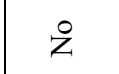 & $z$ \\
\hline & & & & 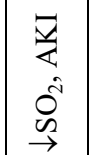 & & $\begin{array}{l}\vec{y} \\
\stackrel{0}{0} \\
\stackrel{\mathscr{n}}{\rightarrow}\end{array}$ & 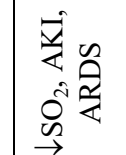 & 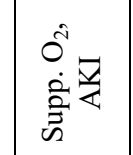 & 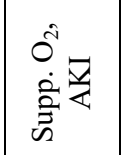 & & $\frac{\nabla}{4}$ & & & & \\
\hline 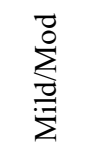 & 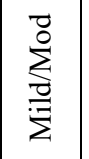 & $\sum_{\substack{0\\
}}^{0}$ & 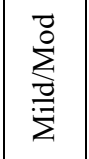 & 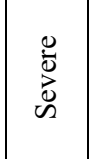 & 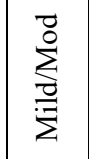 & 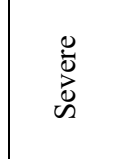 & $\begin{array}{l}\stackrel{D}{D} \\
\stackrel{D}{D}\end{array}$ & $\begin{array}{l}0 \\
\dot{D} \\
\dot{D} \\
\mathscr{n}\end{array}$ & 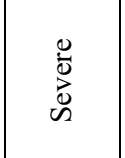 & $\sum_{\substack{z \\
z}}^{\frac{D}{0}}$ & $\begin{array}{l}0 \\
\stackrel{0}{0} \\
\grave{\Delta} \\
\mathscr{n}\end{array}$ & 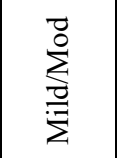 & $\frac{\sum_{0}^{D}}{\sum}$ & $\frac{\sum_{j}^{0}}{\sum_{i}^{\prime}}$ & $\frac{\overrightarrow{0}}{\sum_{0}^{\circ}}$ \\
\hline z & $\tilde{z}$ & $\stackrel{\circ}{z}$ & $\stackrel{\circ}{z}$ & z & $\stackrel{\circ}{z}$ & z & z & $\overbrace{\infty}^{\infty}$ & $\tilde{z}$ & $\tilde{z}$ & $\tilde{z}$ & $\tilde{z}$ & $\bar{z}$ & $\tilde{z}$ & $\tilde{z}$ \\
\hline 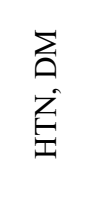 & 量 & $\theta$ & 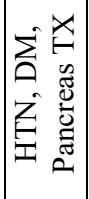 & 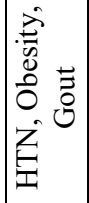 & 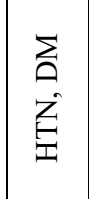 & 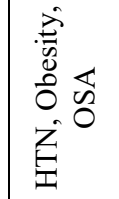 & 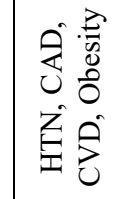 & $\begin{array}{l}\text { 主 } \\
Z \\
\text { Z }\end{array}$ & $\begin{array}{l}\sum \\
\text { 至 } \\
\text { 它 }\end{array}$ & $\tilde{z}$ & $\mathbb{U}$ & 貟 & $\tilde{z}$ & $\begin{array}{l}\sum \\
Z \\
Z \\
Z\end{array}$ & $\tilde{z}$ \\
\hline$\checkmark$ & 点 & $\begin{array}{ll}i & \hat{4} \\
0 & 0 \\
0 & 0\end{array}$ & $\begin{array}{l}\vec{v} \\
\overrightarrow{3} \\
m\end{array}$ & $\begin{array}{l}5 \\
\frac{5}{6} \\
0^{\circ}\end{array}$ & $\begin{array}{l}\overrightarrow{\mathrm{a}} \\
\overrightarrow{\mathrm{O}} \\
-\end{array}$ & $\begin{array}{l}\stackrel{5}{0} \\
\stackrel{0}{0}\end{array}$ & 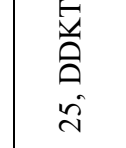 & $\begin{array}{l}\overrightarrow{5} \\
\overrightarrow{3} \\
i\end{array}$ & $N$ & $\begin{array}{l}\stackrel{5}{y} \\
\stackrel{5}{0}\end{array}$ & 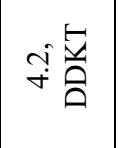 & $\stackrel{\infty}{0} \stackrel{5}{0}$ & $\rightrightarrows \overline{\frac{a}{a}}$ & ì & $\tilde{z}$ \\
\hline$\Sigma$ & $\Sigma$ & $\Sigma$ & $\amalg$ & $\Sigma$ & $\Sigma$ & $\Sigma$ & 山 & $\Sigma$ & $\Sigma$ & $\Sigma$ & $\Sigma$ & 山 & $\Sigma$ & $\Sigma$ & $\Sigma$ \\
\hline in & $\ddot{m}$ & ? & $m$ & $\hat{m}$ & $i$ & 8 & 8 & $\infty$ & gे & $\underset{m}{\infty}$ & t & $\hat{n}$ & ร & $\stackrel{\infty}{n}$ & gे \\
\hline 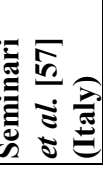 & \multicolumn{2}{|c|}{ 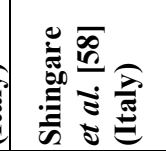 } & \multicolumn{5}{|c|}{ 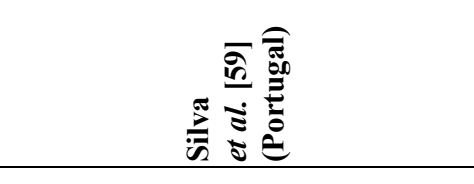 } & 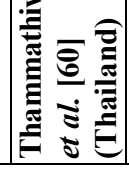 & 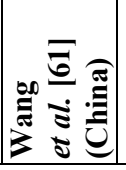 & \multicolumn{5}{|c|}{ 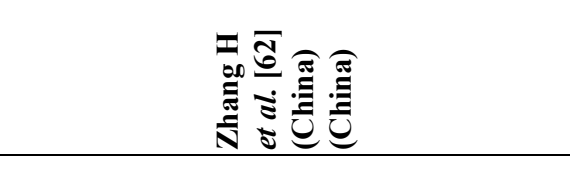 } & 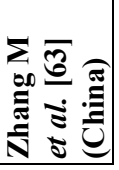 \\
\hline
\end{tabular}




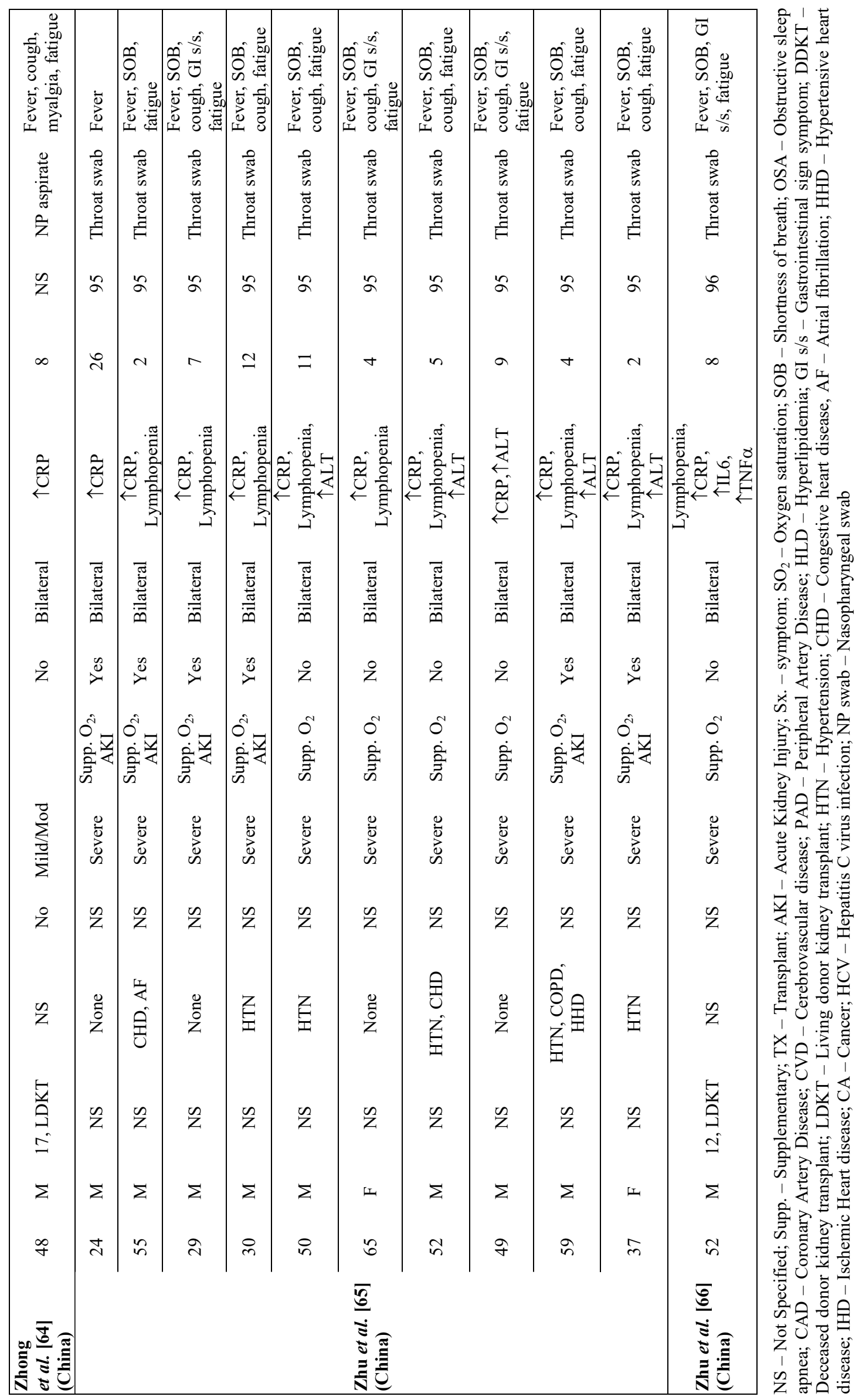




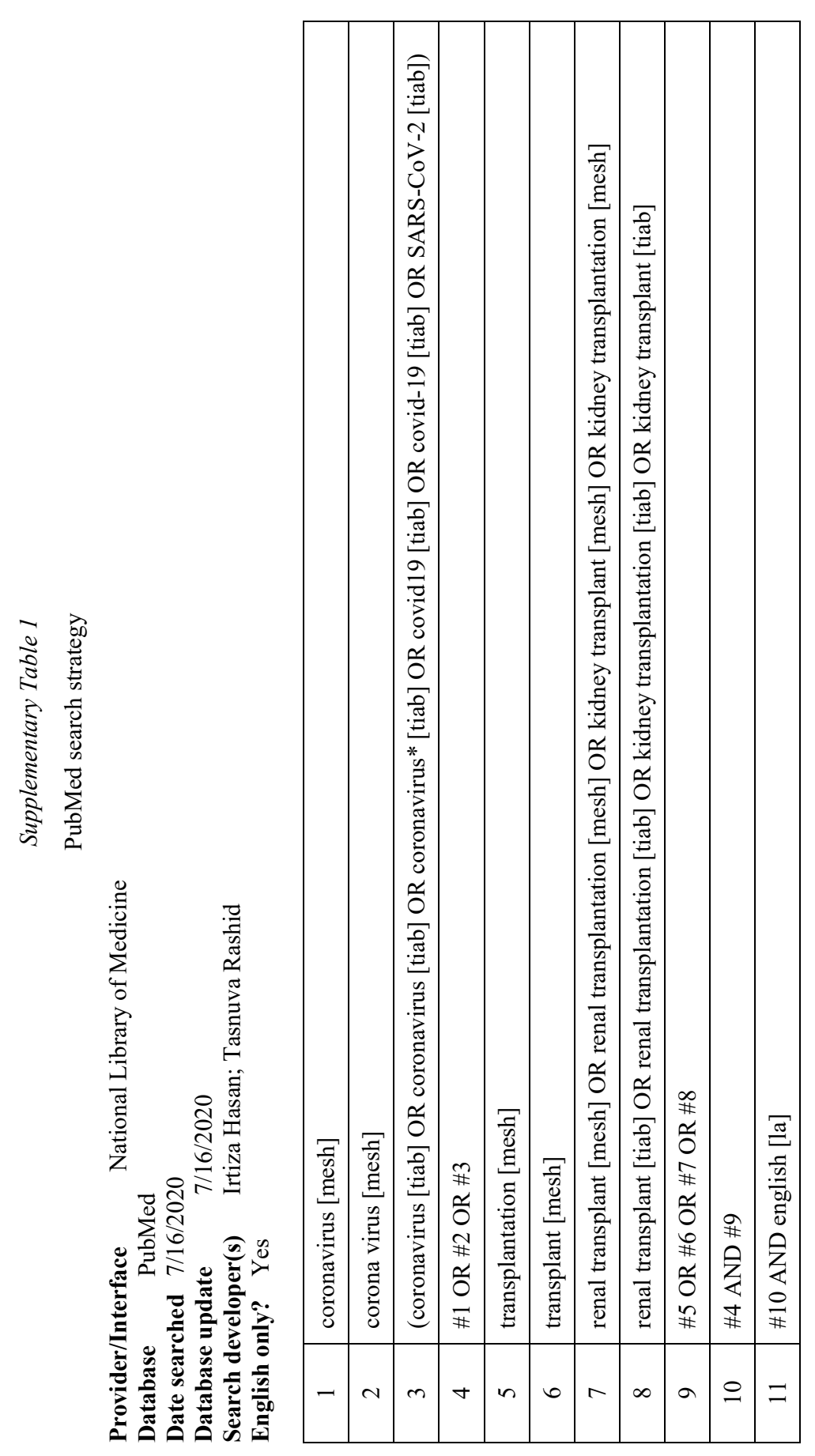




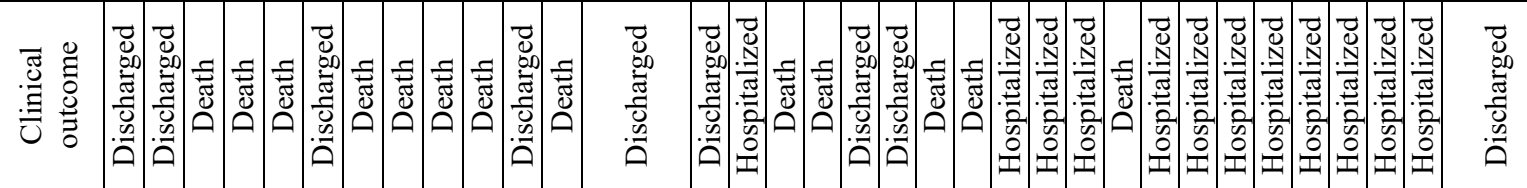

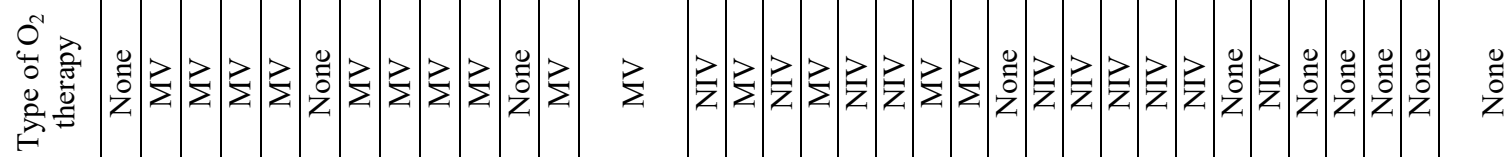

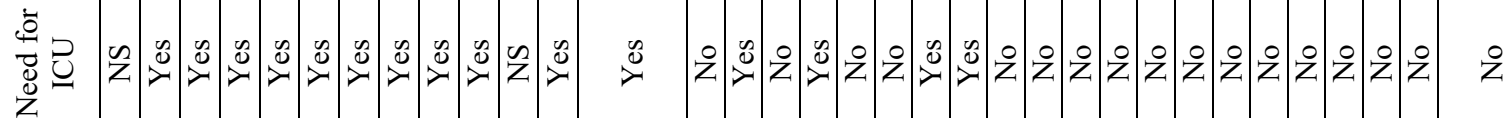
$\frac{n}{n}$

in

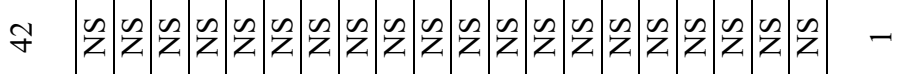

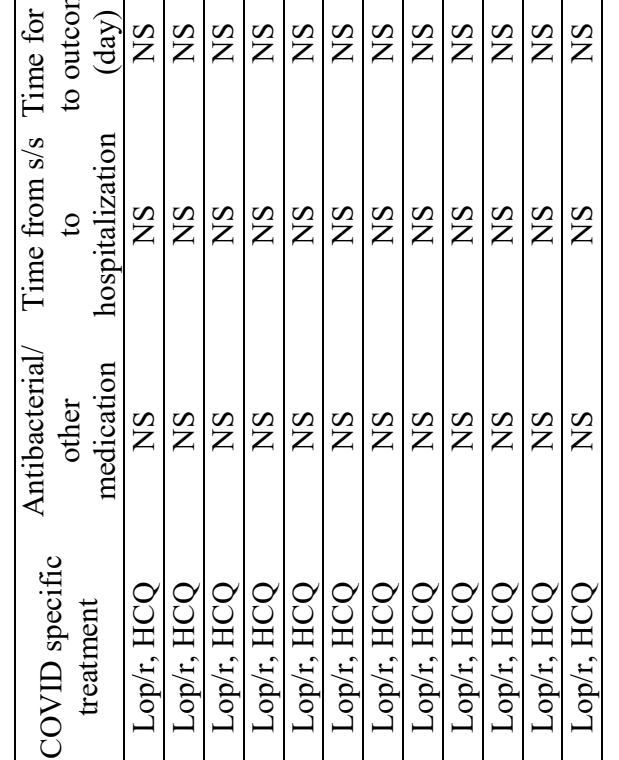

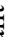

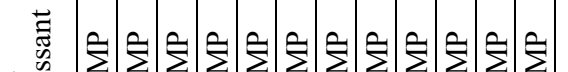

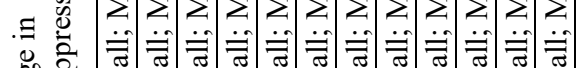

总

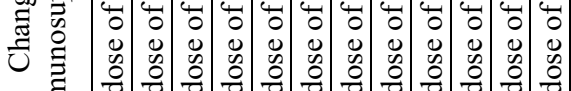

ह

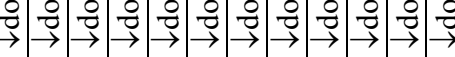

壹

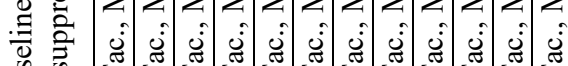

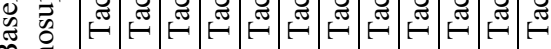

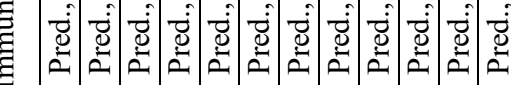

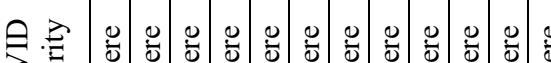

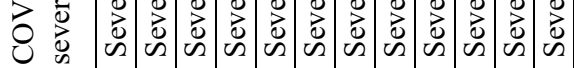

造

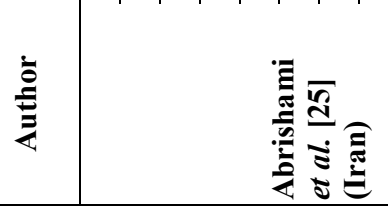

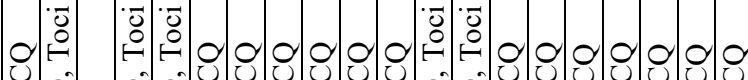
I 0 o

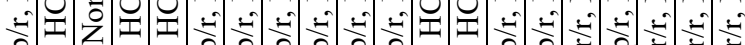

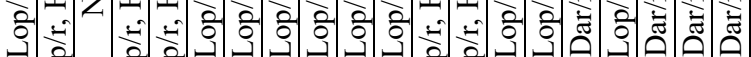
일

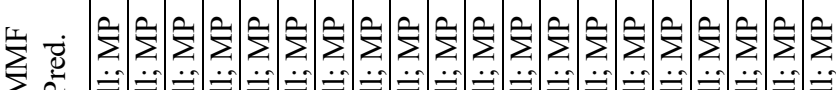

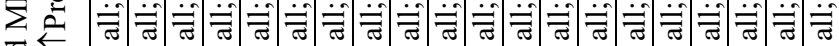

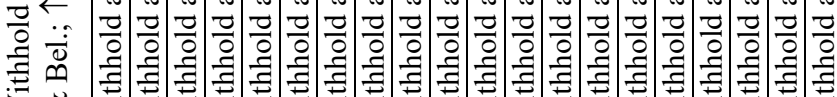

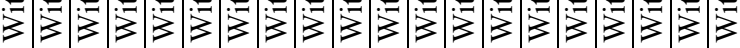
$\sum$ L

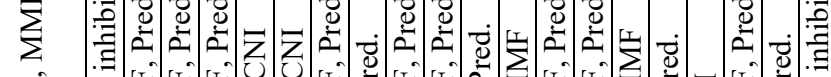
i i

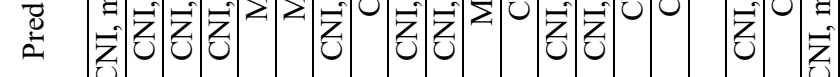

:

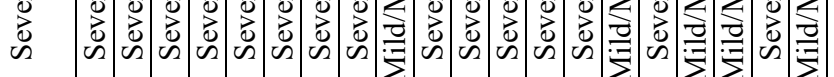
$\sum_{\substack{0 \\ \hline}}^{0}$

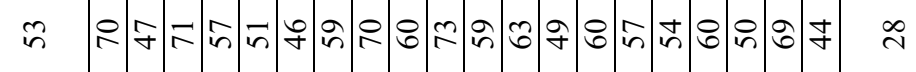
造 


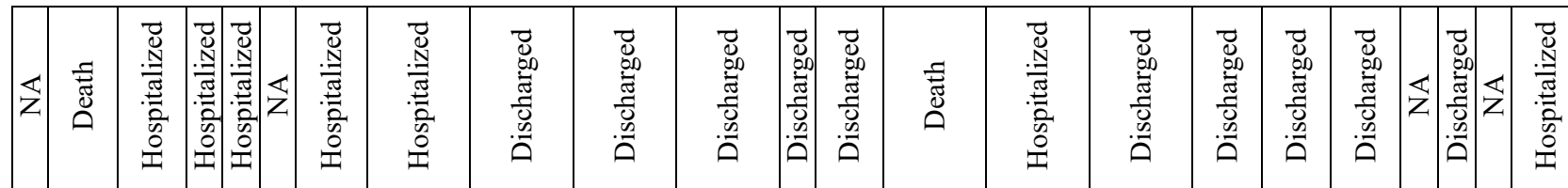

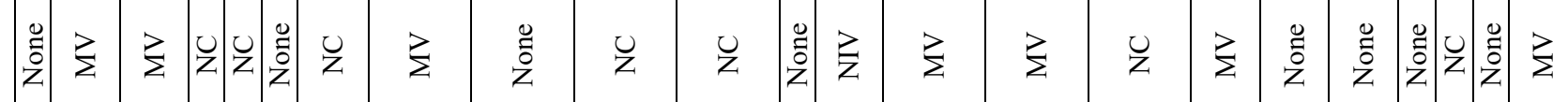

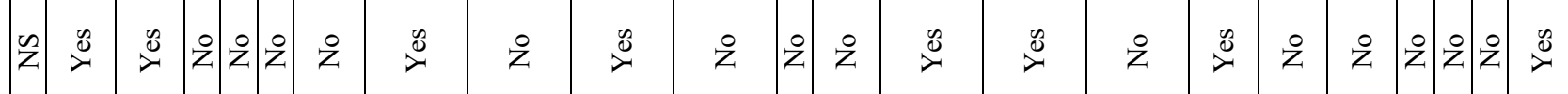

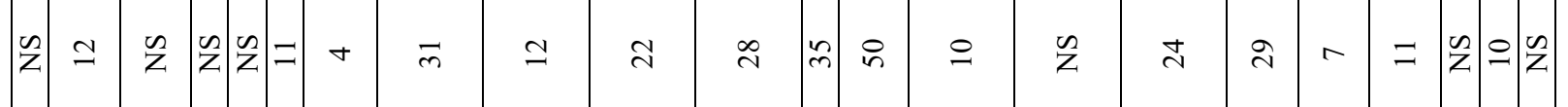

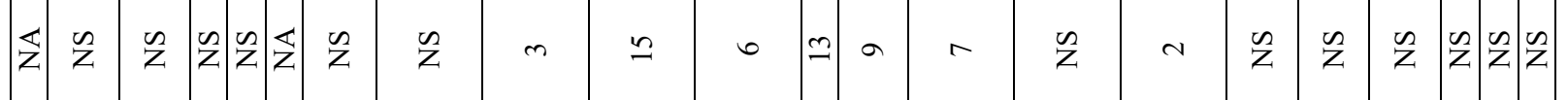

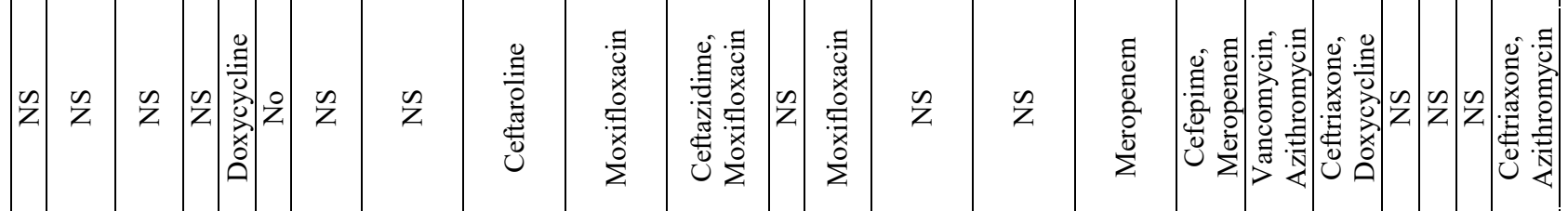

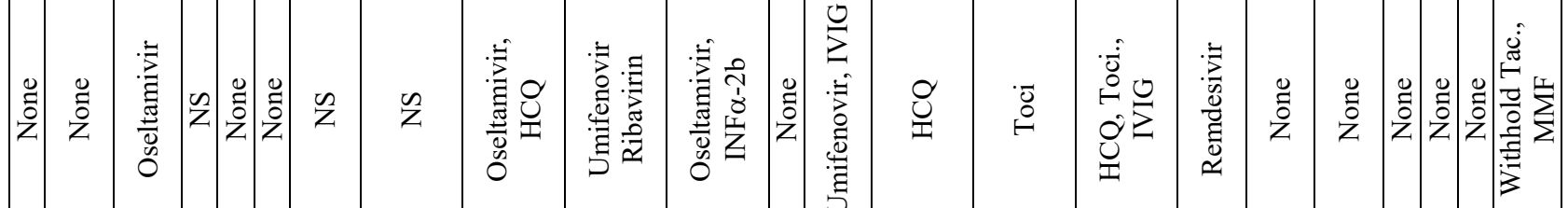

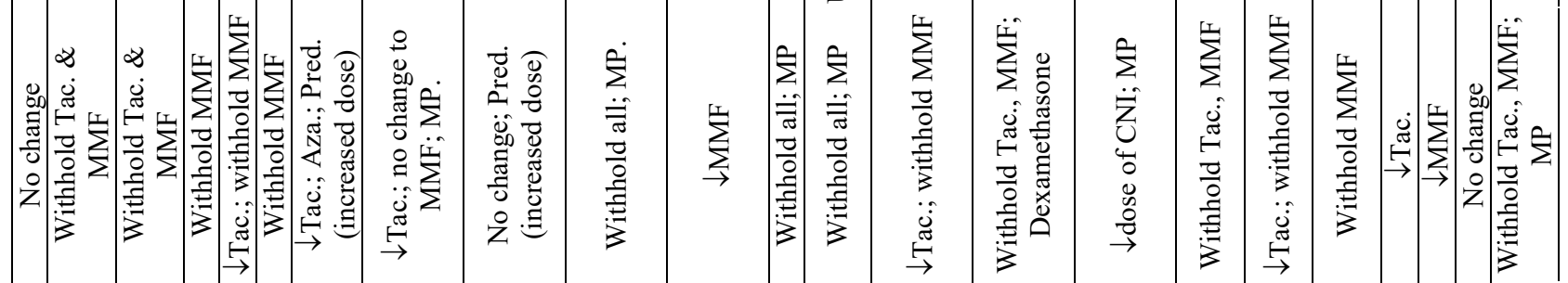

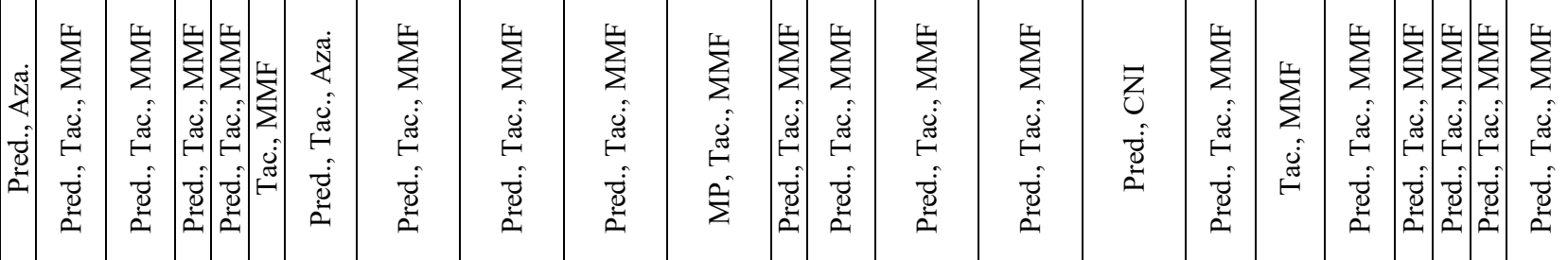

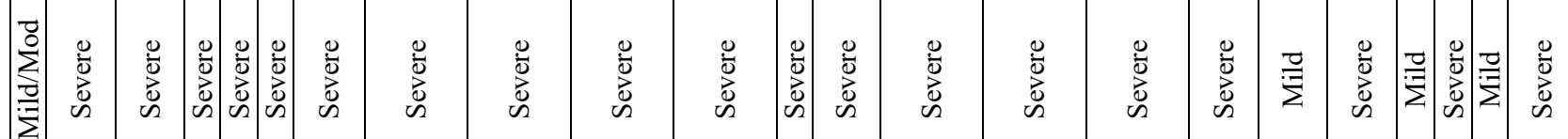
क क

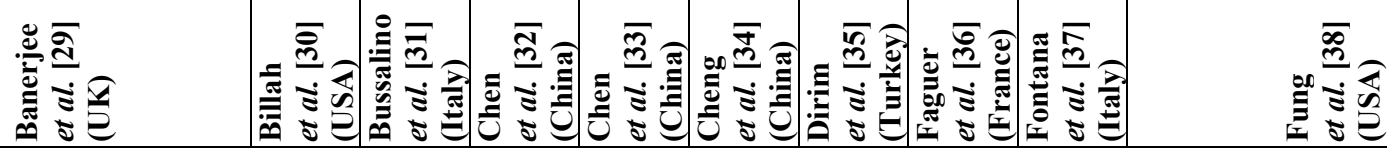




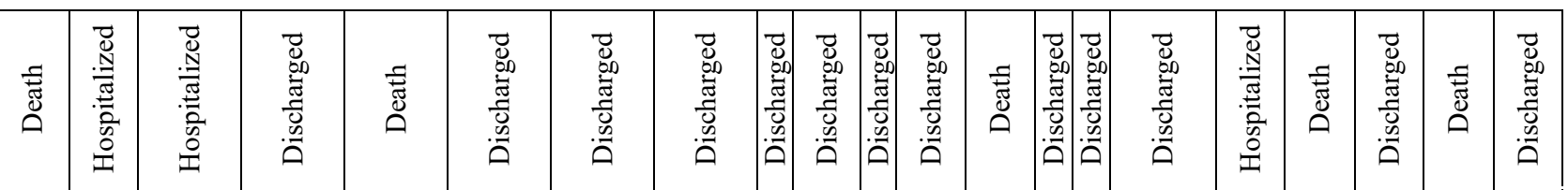

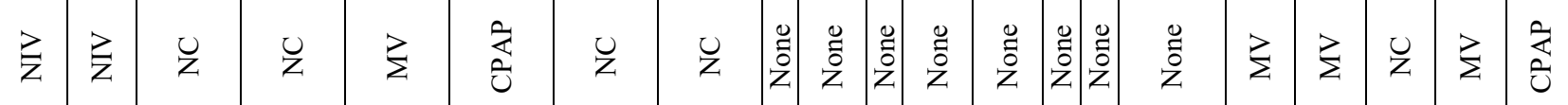

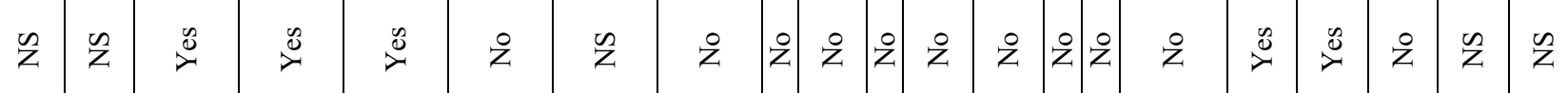

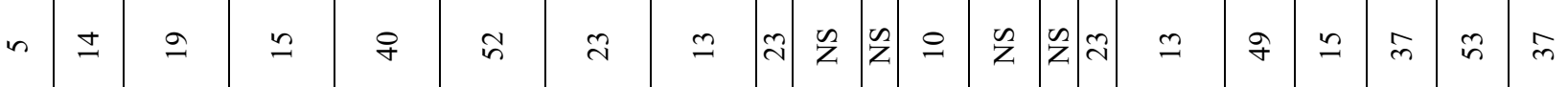

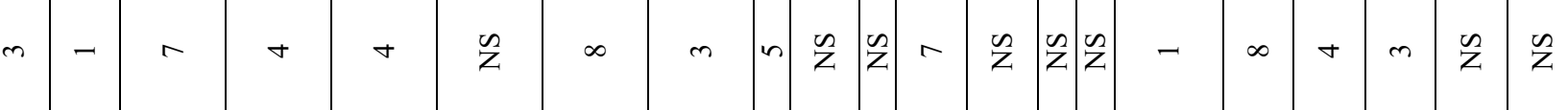

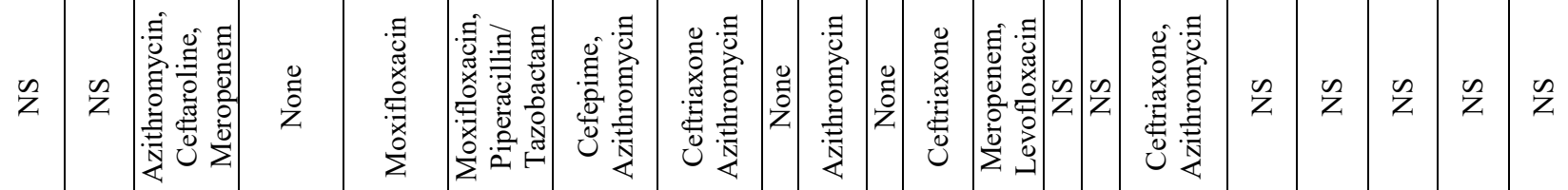

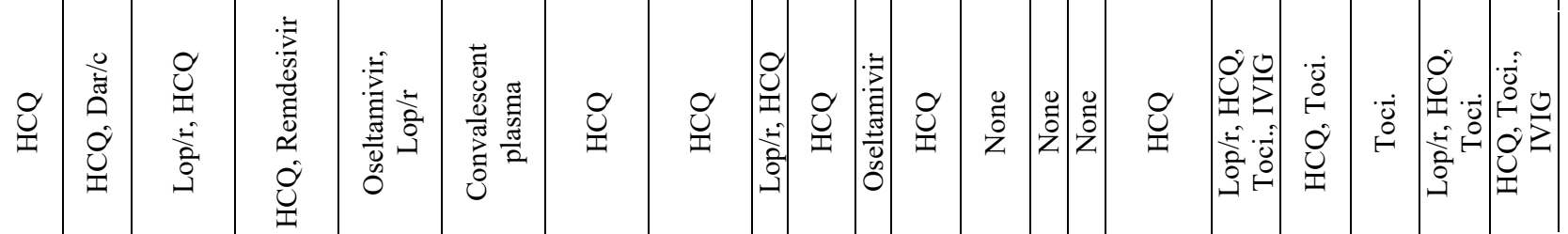

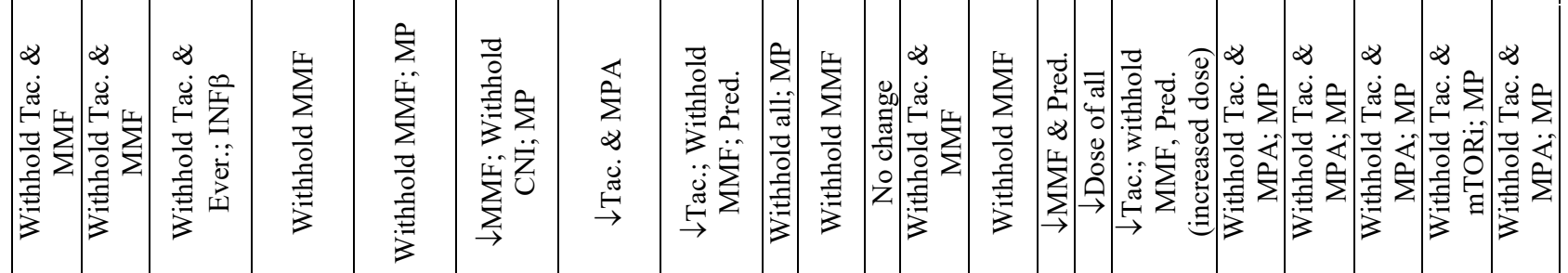

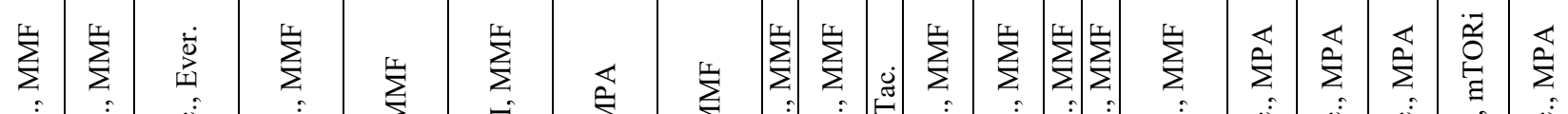

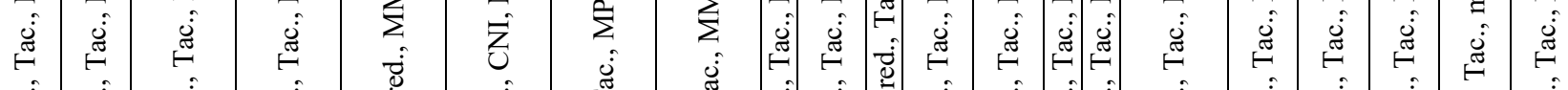
离离离离

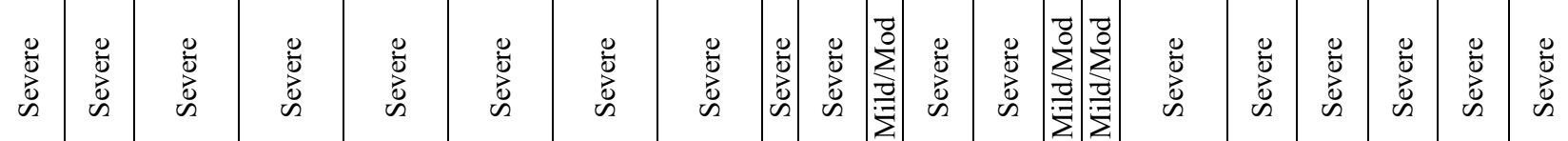

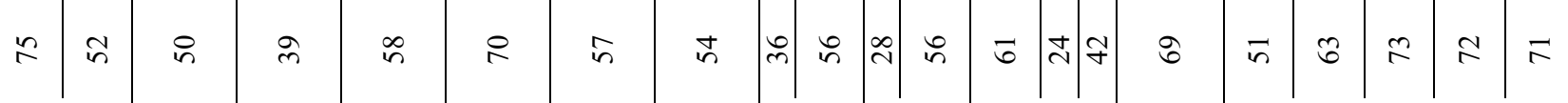

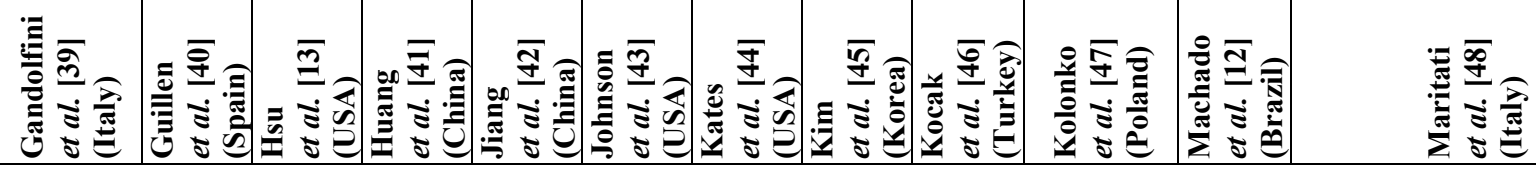




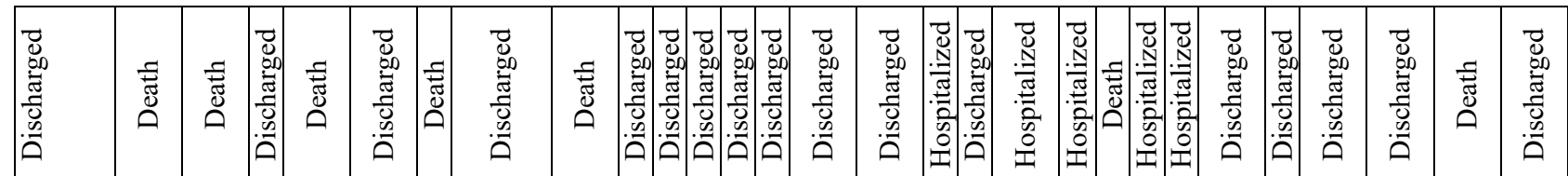

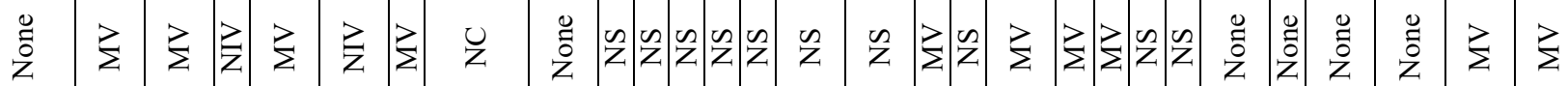

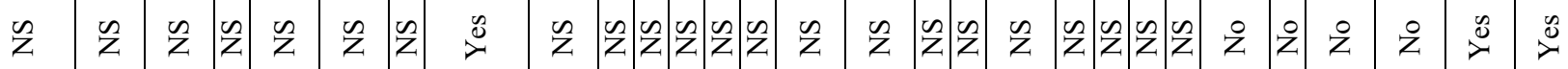

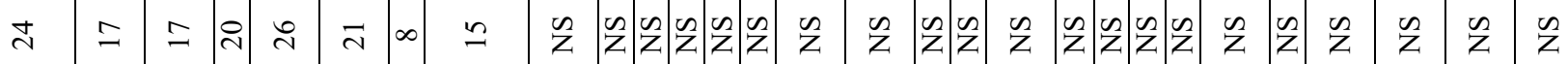

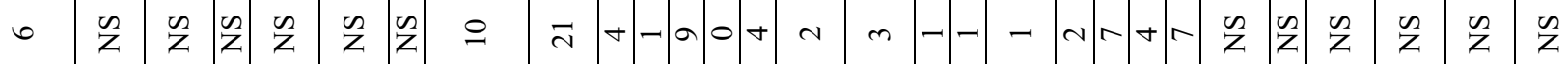

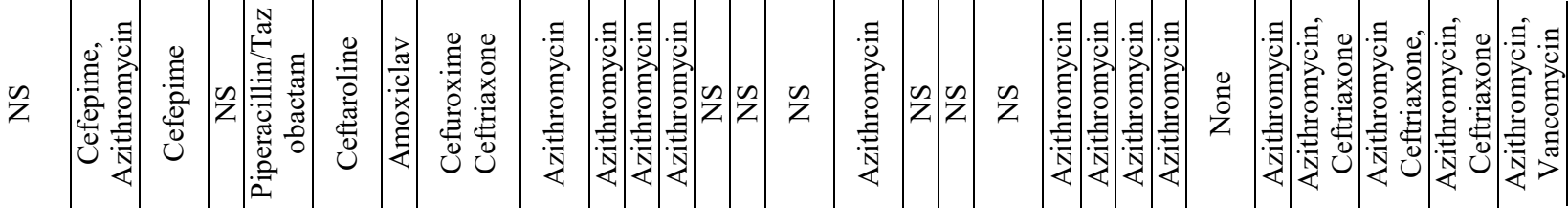

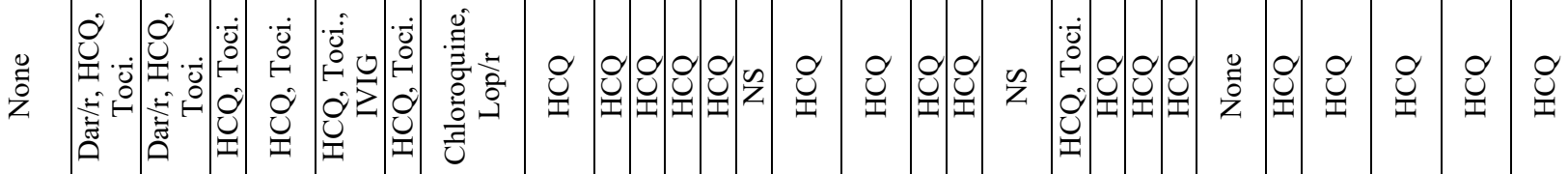

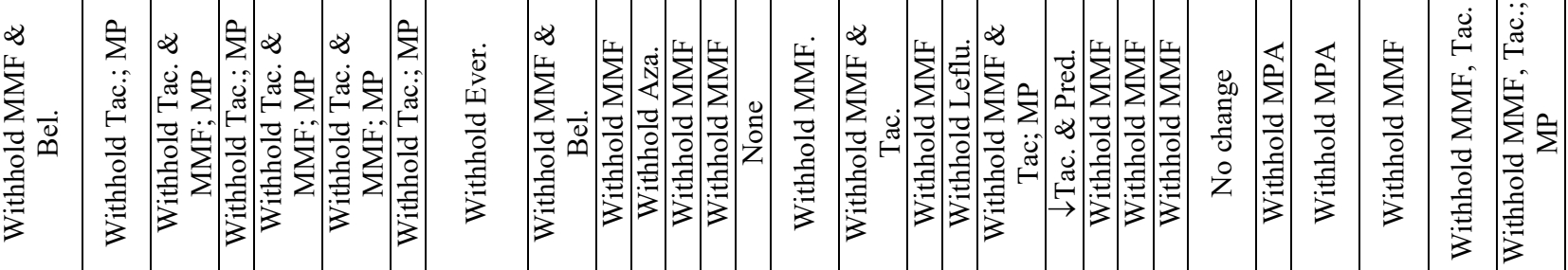

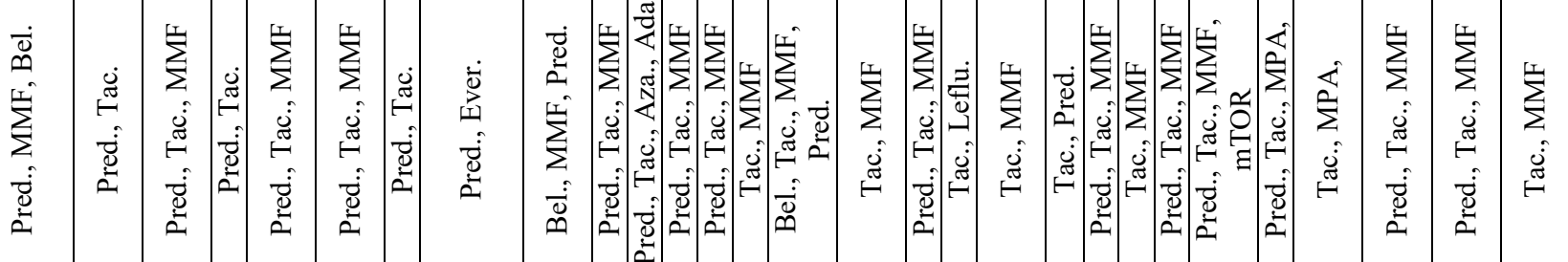

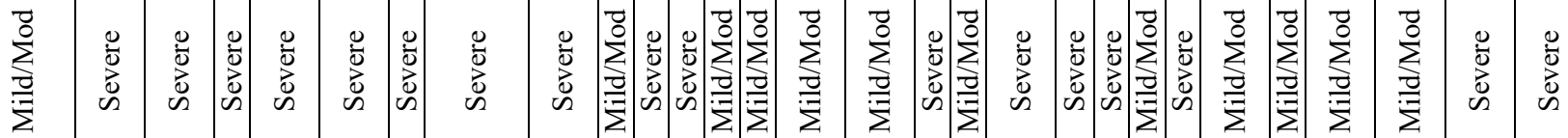

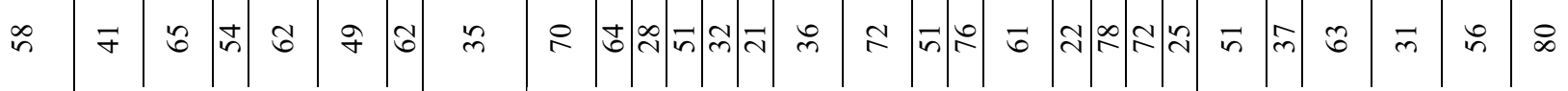

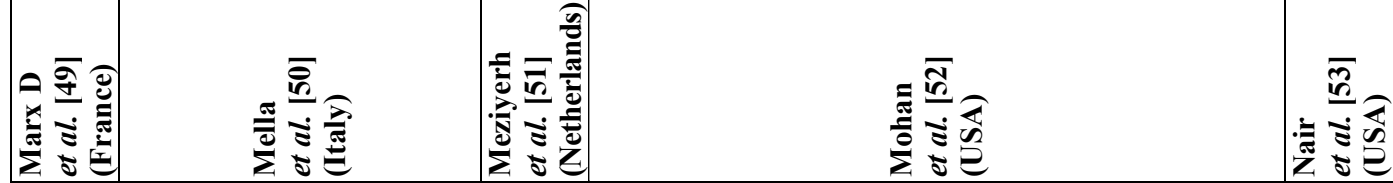




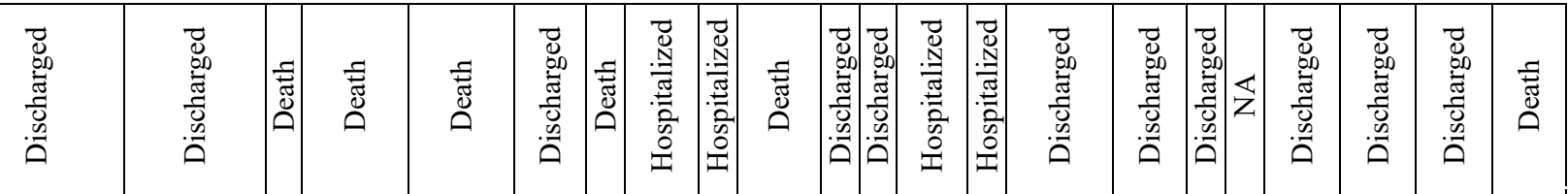

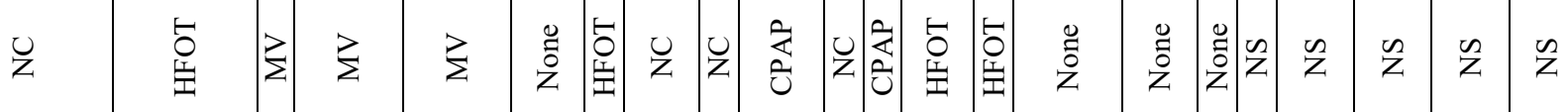

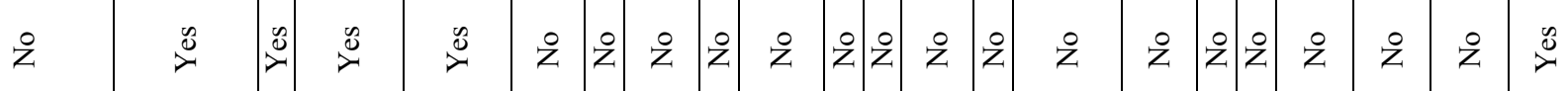

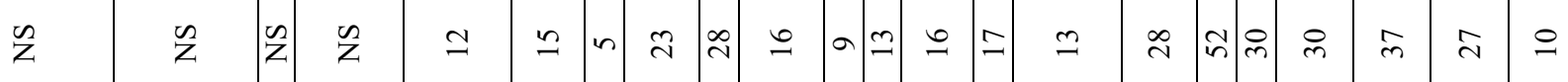

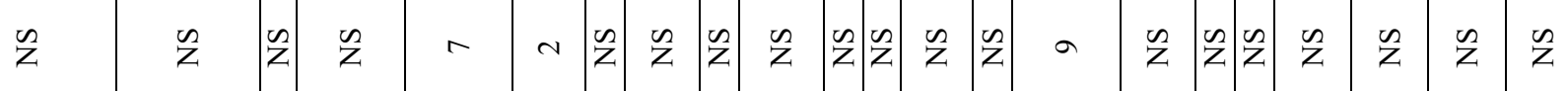

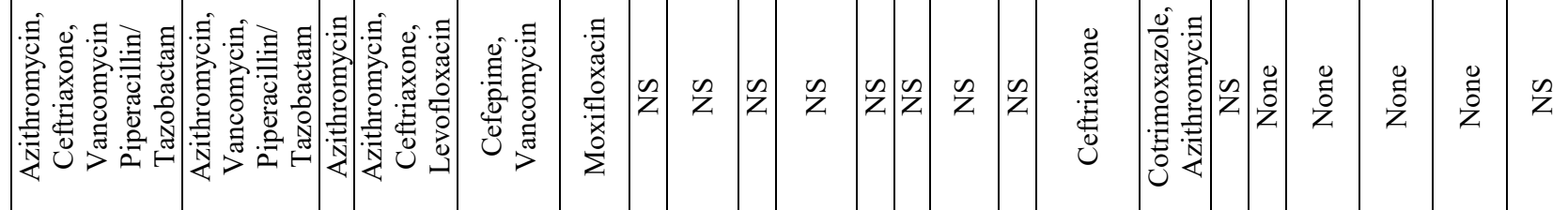

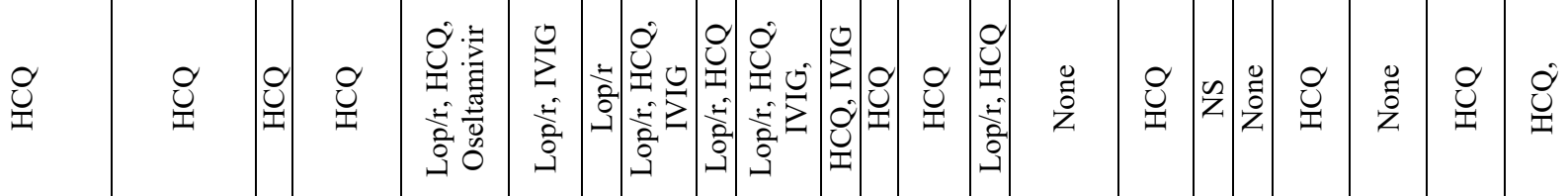

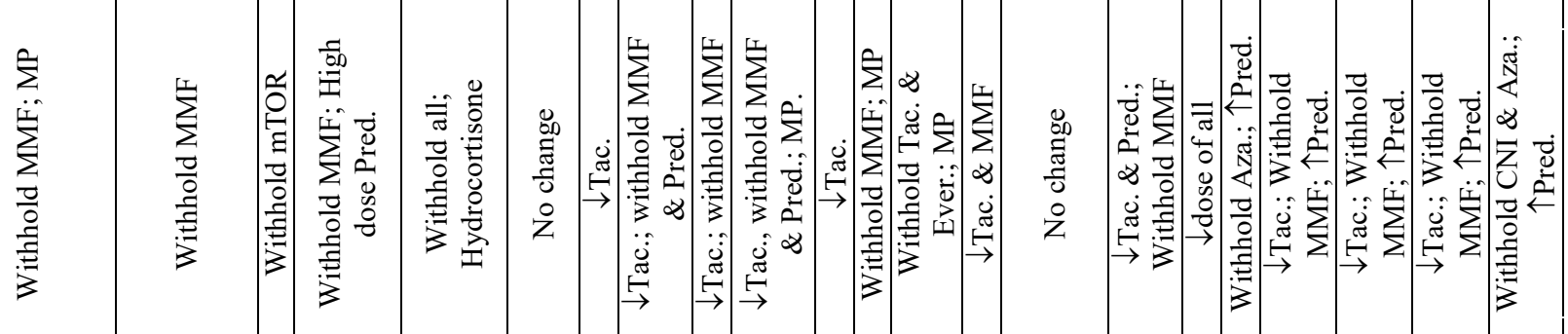

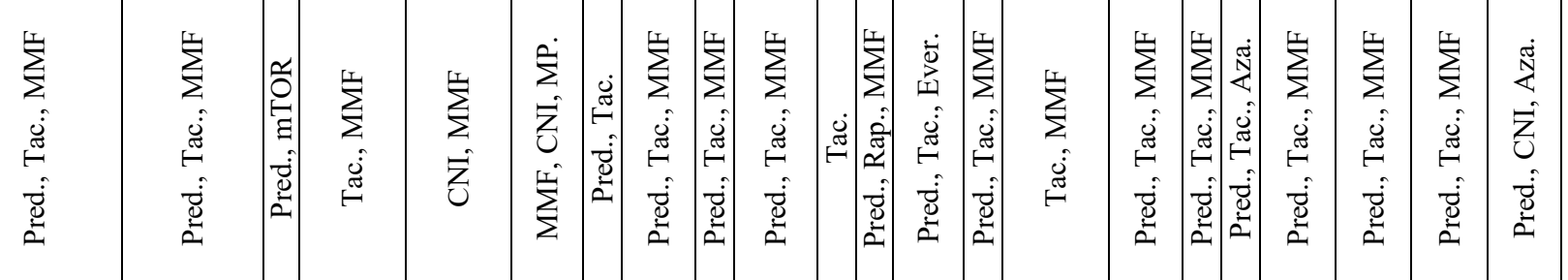

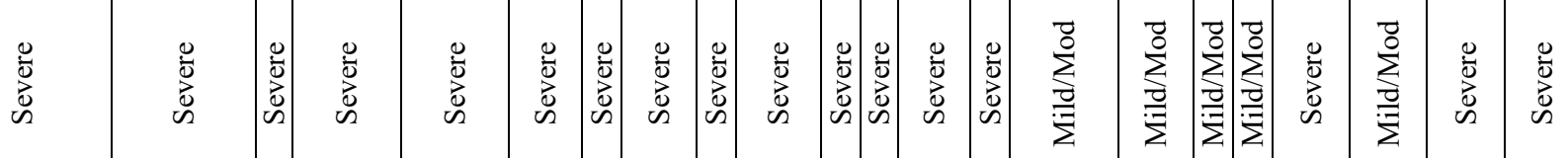

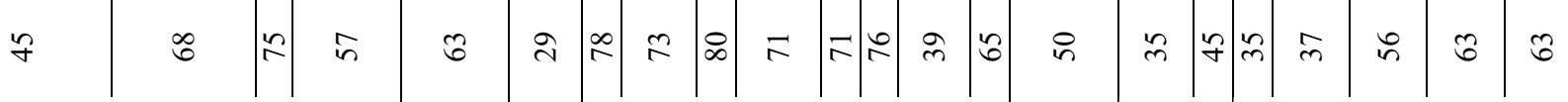

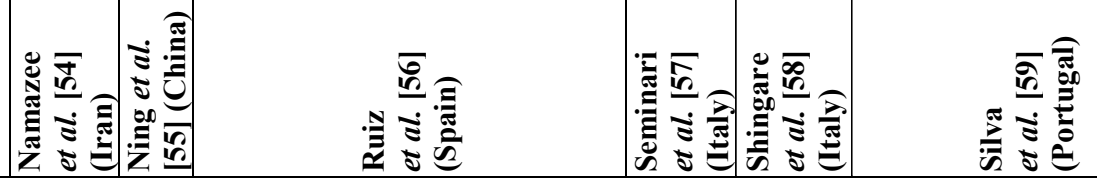




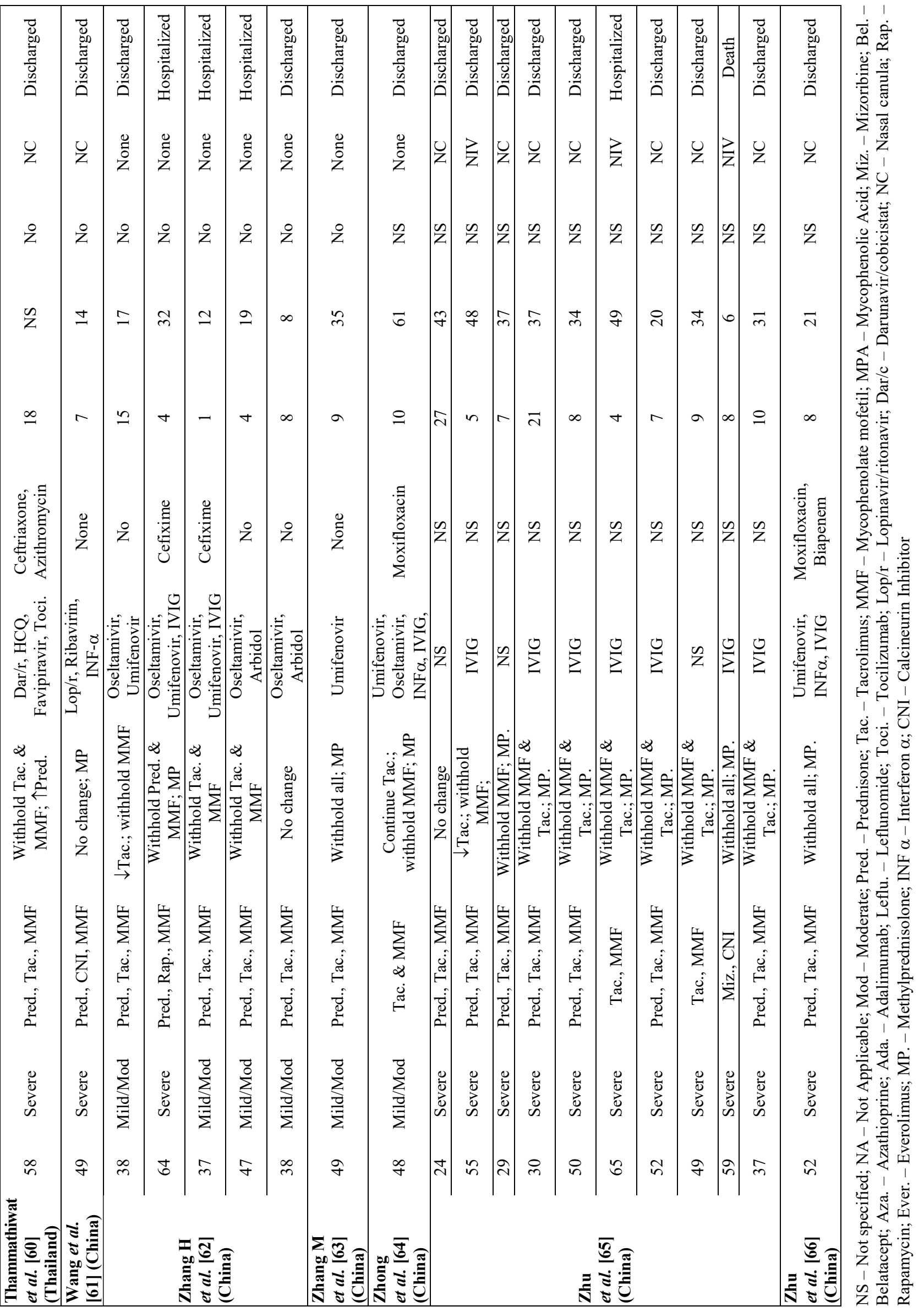




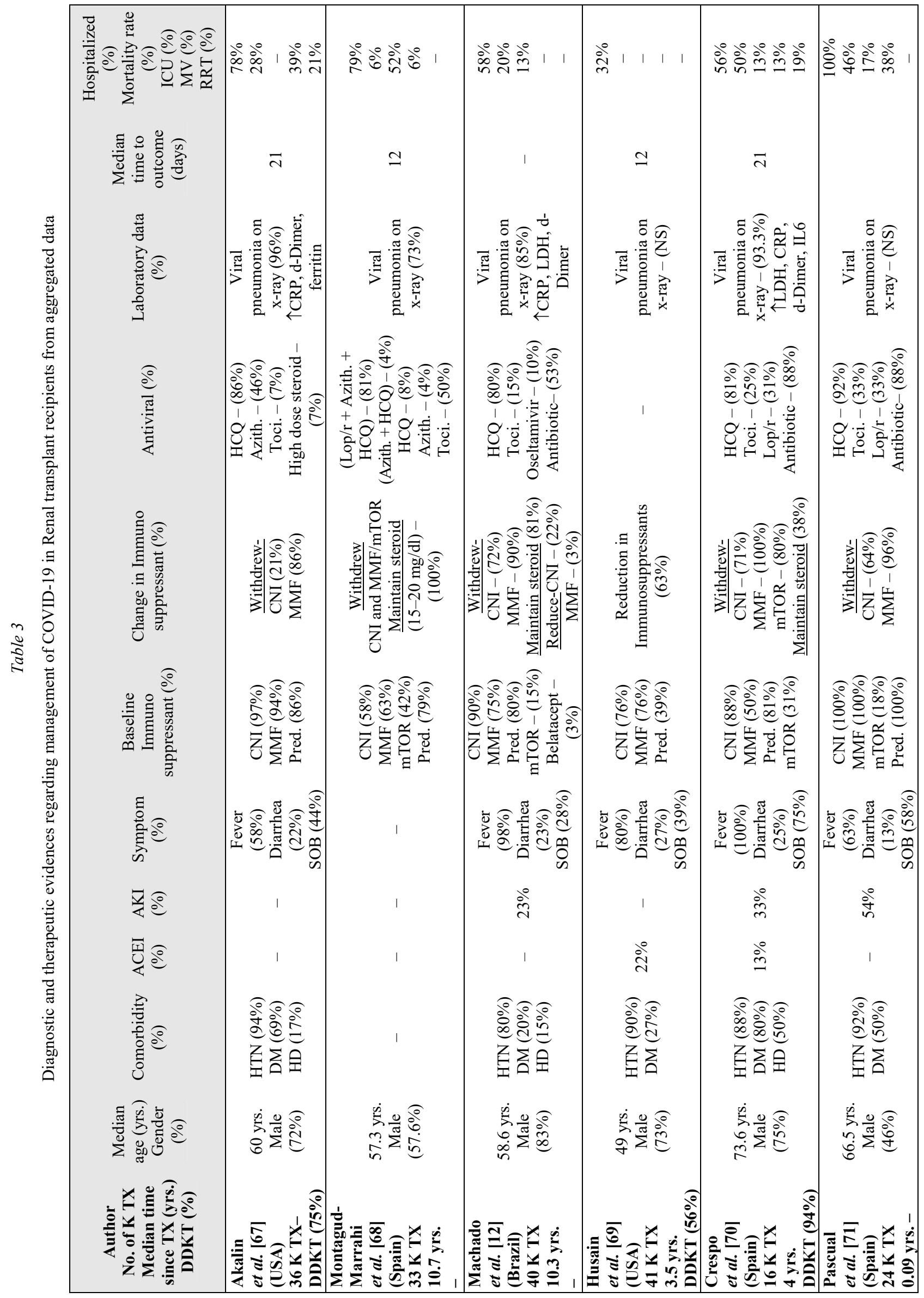




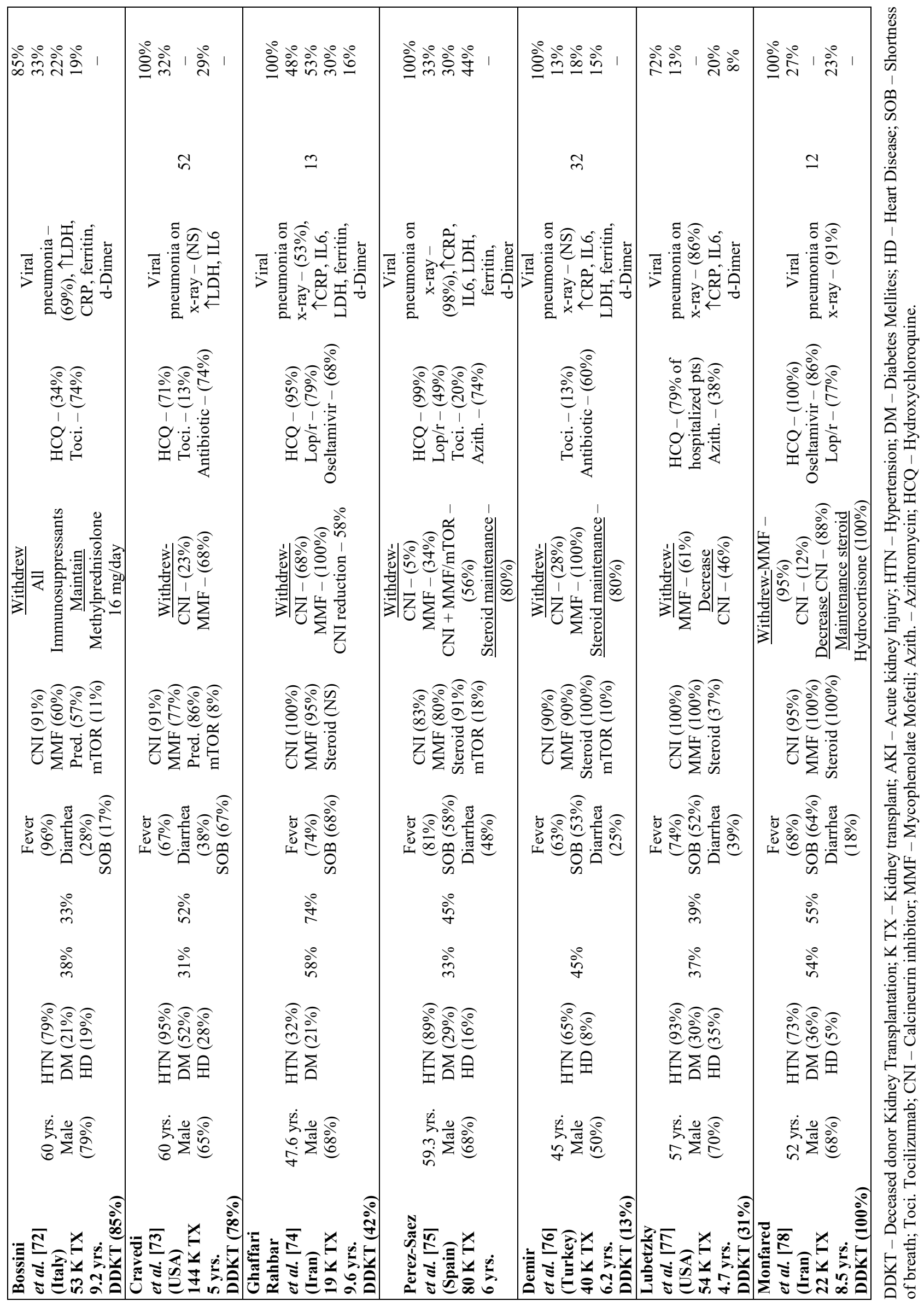


Table 4

Demographics and clinical data for COVID19 infection in renal transplant recipients in relation to disease severity

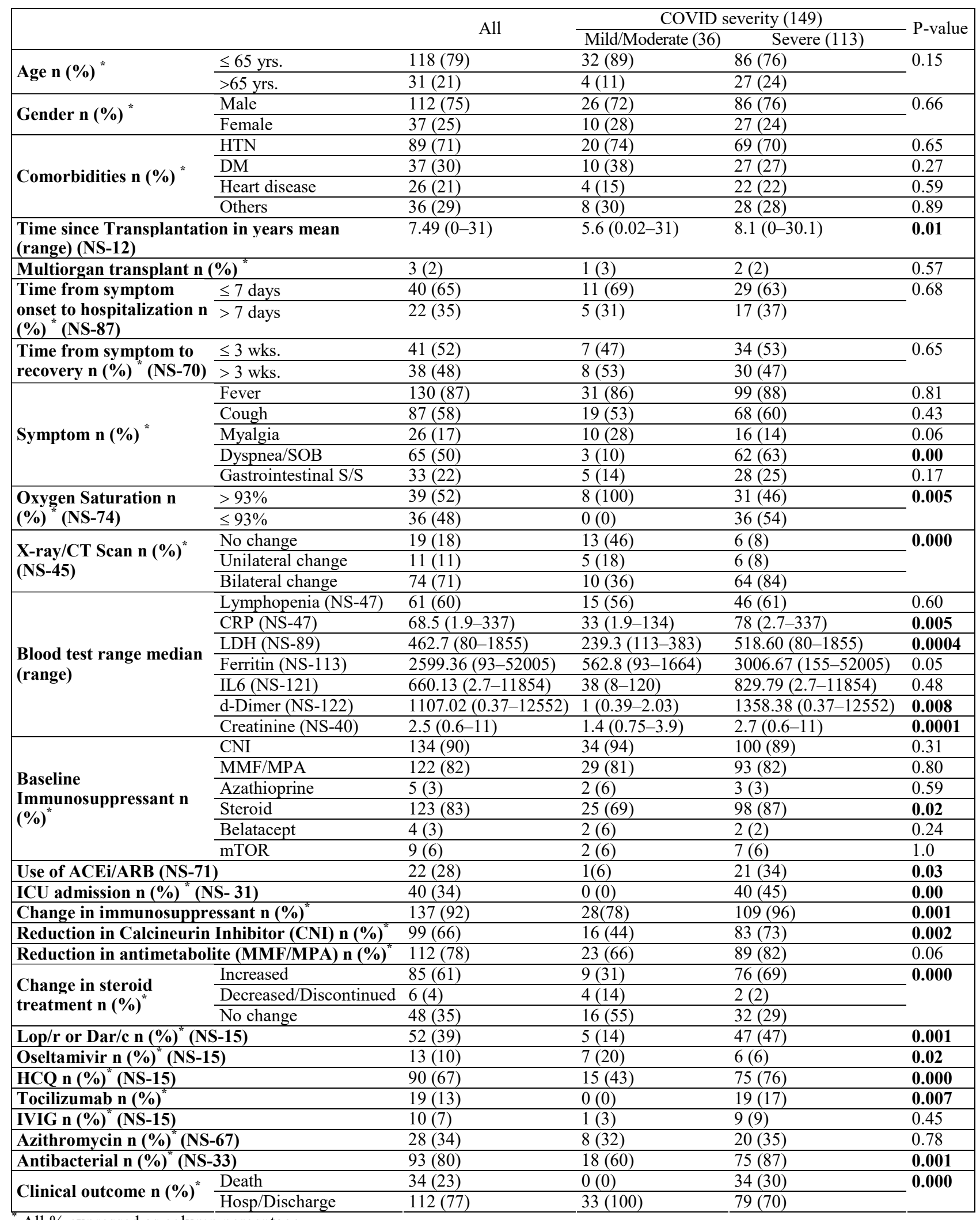

All \% expressed as column percentage

NS - Not specified; HTN - Hypertension; DM - Diabetes mellitus; SOB: Shortness of breath; CRP - C reactive protein; LDH Lactate dehydrogenase; IL 6 - Interleukin 6; MMF - Mycophenolate Mofetil; MPA - Mycophenolic Acid; Lop/r Lopinavir/ritonavir; Dar/c - Darunavir/cobicistat; HCQ - Hydroxychloroquine; IVIG - Intravenous Immunoglobulin; CNI Calcineurin inhibitor (Tacrolimus and Cyclosporine). 


\section{Immunosuppressant and antiviral management}

\section{CHARACTERISTICS AND FACTORS AFFECTING COVID-19 OUTCOME (Table 5)}

Outcome was reported on 144 cases of whom $74(51 \%)$ were discharged, 36 (25\%) remained hospitalized with improved condition and 34 (23\%) patients did not survive the disease. All fatalities occurred in those who had severe disease. When stratified by continent, the fatality rate was numerically highest in Europe (27\%) compared to Asia (26\%) and America (14\%). Table 5 summarizes patients' demographics, clinical presentation and management received by disease outcome.

\section{Demographic and clinical factors}

Compared to patients who were either discharged or remained hospitalized, patients who died had longer duration from transplant to disease diagnosis $(\mathrm{P}=0.03)$ and all had severe disease $(\mathrm{P}=0.001)$. Oxygen saturation $\leq 93 \%$ was more common in those who died than those who remained hospitalized ( $\mathrm{P}=0.00)$ while gastrointestinal symptoms were more common in those who recovered $(\mathrm{P}=0.05)$. Interestingly, presence of other comorbidities, AKI development and $\mathrm{ACEi} / \mathrm{ARB}$ use did not impact overall disease outcome.

\section{Laboratory and immunological factors}

Among various immunological biomarkers reported, only LDH level correlated with outcome. LDH was elevated in $68 \%$ of those who died as compared to $29 \%$ in those who were either hospitalized or discharged $(p=0.001)$. The significance was still maintained when LDH level was classified as $\leq 300 \mathrm{U} / \mathrm{L}$ and $>300 \mathrm{U} / \mathrm{L}$ with majority deaths $(95 \%)$ occurring in patients with $\mathrm{LDH}$ level $>300 \mathrm{mg} / \mathrm{dl}$ $(p=0.001)$. A box plot for different immunological markers and serum creatinine stratified by disease severity and disease outcome is outlined in Figure 3.

\section{Post-hospitalization management}

Overall change in immunosuppression was more aggressive in those who died or remained hospitalized $(\mathrm{P}=0.002)$. Immunosuppression change was mainly driven by reducing or eliminating CNI in $79 \%$ and $86 \%$ in deceased and hospitalized patients, respectively, compared to only $54 \%$ in patients who were discharged $(\mathrm{P}=0.001)$. Steroid monotherapy was also more common in those who died $(56 \%)$ or remained hospitalized $(69 \%)$ compared to only $38 \%$ in those who were discharged $(\mathrm{P}=0.01)$. The antiviral agents Lop/r or Dar/c, HCQ and antibacterial agents were more commonly used in those who did not survive the disease or required prolong hospitalization. ( $\mathrm{P}<0.05$ for both). There was no difference in oseltamivir, azithromycin, tocilizumab or IVIG utilization among the 3 outcome groups. The majority of the patients who died required ICU support (78\%) and non-invasive ventilation or mechanical ventilation $(\mathrm{P}<0.001)$.

\section{THERAPEUTIC MANAGEMENT AND DISEASE SURVIVAL}

We then divided the 144 cases with reported outcome into survivors and non-survivors to determine if there was any difference in survival according to the different strategies of immunosuppression modification or antiviral combinations. As demonstrated in Table 6, more aggressive reduction in immunosuppression was undertaken in non-survivors $(26 \%$ vs $12 \%$; $\mathrm{p}=0.03$ ). Corticosteroid dose increase or IV methylprednisolone use were not associated with survival. There was no significant difference in survival between different antiviral combinations with the exception of higher mortality in those who received Lop/r and HCQ combination $(55 \%$ vs $30 \% \%, \mathrm{P}=0.02)$ and HCQ at a dosage of $400 \mathrm{mg}$ once daily ( $44 \%$ vs $19 \%, \mathrm{P}=0.04)$.

\section{Effect of time of publication on disease survival}

To explore if disease survival has changed overtime, we analyzed the mortality rate according to the time of publication of each report. We compared survival between early reports (published on or before May $31^{\text {st }}, 2020, \mathrm{n}=29$ studies) and late reports (published from June $1^{\text {st }}$ to July 16 th $2020, n=15$ studies). Overall mortality rate was comparable between the early (23.6\%) and late (23.6\%) reports, Figure 2.

\section{DISCUSSION}

In the current study, we identified demographic, clinical, laboratory, radiological, and treatment specific factors associated with disease severity and mortality in 149 globally representative cases of kidney transplant recipients with COVID-19. Our case series limited to aggregated data on 561 renal transplant recipients 
also reported similar findings. Results indicate that severe disease $(76 \%)$ and mortality $(23 \%)$ were much higher in kidney transplant recipients compared to the general population. According to a review by Patel et al. the mortality rate for hospitalized patients in the general population was found to be around $13 \%$ [14]. We also demonstrated that dyspnea, radiological evidence of bilateral lung involvement, $\mathrm{ACEi} / \mathrm{ARB}$ use, higher LDH, CRP, ferritin and d-Dimer levels were all factors that correlated with disease severity while older recipient age and presence of comorbidities did not. Mortality occurred only in patients with severe disease and was higher in patients older than 65 years, those with longer duration from transplant to disease diagnosis and in those with hypoxia and higher LDH at presentation. Despite the higher rate of AKI, mortality did not relate to AKI development which is in contrast to studies in the general population. Despite aggressive CNI dose reduction and higher utilization of intravenous corticosteroid, antiviral and antibacterial agents in those with severe disease, none of the treatment combinations demonstrated a clear survival advantage. Interestingly, HCQ and Lop/r combination and high once daily dose of HCQ were more commonly utilized in those who did not survive.

In the current study, we used a standardized classification for COVID-19 severity developed by the WHO. We demonstrated that the majority (76\%) of the hospitalized kidney transplant recipients reported, had severe COVID-19 which is in striking contrast to the reported disease severity of $5 \%$ in hospitalized patient in the general population [15]. While under-reporting of mild cases and excluding patients managed in the outpatient setting could have modified the true incidence of COVID-19 severity in the kidney transplant population, this alarming rate of disease severity suggests that close monitoring of kidney transplant recipients who develop COVID-19 should be undertaken especially since all reported disease fatalities were in patients who had severe disease. In spite of differences in severity, the overall presentation of COVID-19 in renal transplant recipients was similar to the pattern observed in the general population.

We identified a slightly different risk factor profile for COVID-19 severity in kidney transplant recipients compared to the general population. While studies in the general population indicated that age, gender, and comorbidities are prognostic factors for disease severity, the current study demonstrated that none of these factors correlated with disease severity in the kidney transplant recipients $[16,17]$.
However, symptomatology especially dyspnea, and radiological evidence of bilateral lung involvement at presentation were factors associated with disease severity in this group of patients. Similar to studies of COVID-19 in the general population, we found increased inflammatory markers especially elevated CRP, LDH, Ferritin and d-Dimer levels to be associated with disease severity in the hospitalized kidney transplant recipients and therefore these markers should be monitored at presentation of any COVID-19 patients to identify those patients at risk of developing severe COVID-19 [6] [18]. Although serum creatinine was higher in those with severe disease, this was probably related to including AKI as defining criteria of disease severity. One important observation is the high rate of AKI in kidney transplant recipients. In contrast to reports from hospitalized COVID-19 cases in the general population where the incidence of AKI was found to be between 4 and $10 \%$, the incidence of AKI in hospitalized kidney transplant recipients was much higher at 49\% [19]. Interestingly, ACEi/ARB were more commonly in use in cases who developed severe disease. The reason for the association between ACEi/ARB uses and disease severity is unclear but could be related to higher rates of AKI and hence severe disease with ACEi/ARB use. Since we could not perform a multivariate analysis due to the small number of cases, the association between ACEi/ARB and disease severity in kidney transplant recipients should be further explored in future studies.

Reported COVID-19 fatality rate in the general population ranged between $2 \%$ and $15 \%$ and varied according to geographical location. In the US, the case fatality rate was found to be $1.7 \%$ with variation by county $[20,21]$. A study by Buckner et al. reporting the clinical feature and outcome of COVID-19 in hospitalized general population in Seattle region identified a high case fatality rate of $33 \%$ in non-kidney transplant recipients but the older age and the multiple comorbidies of the study participants explain these results [22]. In contrast, the current study demonstrated that the fatality rate in the hospitalized kidney transplant recipients was much higher at $23 \%$ and remained relatively stable overtime (Figure 2) which is not surprising giving the higher observed rate of severe disease in this group of patients. Factors associated with disease fatality in kidney transplant recipients were also slightly different from the general population. While AKI was associated with high mortality in the general population, AKI was not found to correlate with fatality in kidney transplant patients 
$[19,23]$. Reason for the difference of the impact of AKI on outcome between the general and the kidney transplant populations is unclear and needs further investigation. Longer duration from transplant to disease diagnosis was associated with worse survival. Although this could be related to other confounders such as older patients' age, this finding still implies that kidney transplant patients are at risk of dying after acquiring COVID-19 even if they were transplanted many years prior to disease diagnosis. Compared to studies in the general population that showed that CRP, IL-6, and ferritin to be predictors of mortality, elevated LDH was the only immunological predictor of both severity and worse outcome in kidney transplant patients. The higher observed mortality developed despite aggressive reduction in immunosuppression medication (especially $\mathrm{CNI}$ ), and higher utilization of antiviral agents. It is important to mention that increasing corticosteroid dose or escalation to parenteral methylprednisolone were not associated with survival advantage. While none of the different antiviral combinations were associated with survival advantage, HCQ and Lop/r combination and higher daily HCQ dosage were more commonly utilized in non-survivors. Reason for the higher mortality of these medication combinations is unclear but could be related to higher disease severity which led to the desperate use of these experimental regimens. Irrespective of the cause, the lack of survival advantage of any specific antiviral or antiviral combination along with the stable fatality rate over the last 5 months suggests that the increased fatality rate of COVID-19 in hospitalized kidney transplant patient is probably related to a host of other factors including lack of in vivo efficacy of the available anti-viral agents, lack of drugs targeting COVID-19 virus, virulence of the virus, and disease associated complications including AKI, ARDS and multiorgan failure [24]. One important take home message however was the relative safety of short-term reduction or withdrawal of immunosuppression medications as only 2 patients experienced non-biopsy proven acute rejection.

Although the current study has various strengths, there are some limitations. This study relied on previously published reports to extract the demographic, clinical, laboratory and management related information which was utilized to perform data analysis. In some cases, some of the necessary information was missing which could have affected our results. For a better understanding of COVID-19 there is a need for access to data even if provided in supplementary section or as appendix. Secondly, there is a need for using standard case definition as well as definition of study parameters for generalizability as well as comparability with other studies. A relatively small sample size could have reduced the statistical power to observe different association. Relying on previously published reports has also limited our ability to perform a multivariate analysis to identify independent predictors of disease severity or mortality in COVID-19 infected kidney transplant recipients. We also cannot exclude the possibility of publication bias where milder cases are less likely to be reported. Although we tried to reduce the risk of bias in the study but there is a possibility of presence of inherent clinical heterogeneity which might limit our generalizability. We limited our literature search to July $16^{\text {th }}, 2020$ and did not include any published records after this date. Also, literature focusing on COVID-19 is growing rapidly and hence the rates from this study might change when newer studies are added to the database. Due to these limitations, the results of this study should be viewed as preliminary information. As the disease continues to spread around the globe, large scale multicenter studies are needed to clarify appropriate management strategies of COVID-19 in kidney transplant recipients.

\section{CONCLUSION}

Using a globally representative sample of COVID-19 in 149 hospitalized kidney transplant recipients we conclude that severe COVID-19 is more common in this population affecting $76 \%$ of cases. Although mortality rate was only $23 \%$, mortality in hospitalized kidney transplant recipients was much higher than the reported COVID-19 global mortality rate in the general population. AKI was highly prevalent in kidney transplant patients but it did not correlate with death. Elevated LDH at presentation and longer time since transplantation were associated with both disease severity and mortality. Immunosuppression management and antiviral utilization varied widely among different reports reflecting the lack of consensus on how best to manage these patients. Despite aggressive CNI dose reduction and higher utilization of antiviral and antibacterial agents in those with severe disease, none of the treatment combinations demonstrated a survival advantage including increasing corticosteroid dose or escalation to parenteral methylprednisolone. Lop/r and HCQ combination and high once daily dose of HCQ were more commonly utilized in non-survivors, an association that needs to be further confirmed in future studies. 
Table 5

Factors affecting the outcome of COVID19 infection in hospitalized renal transplant recipients

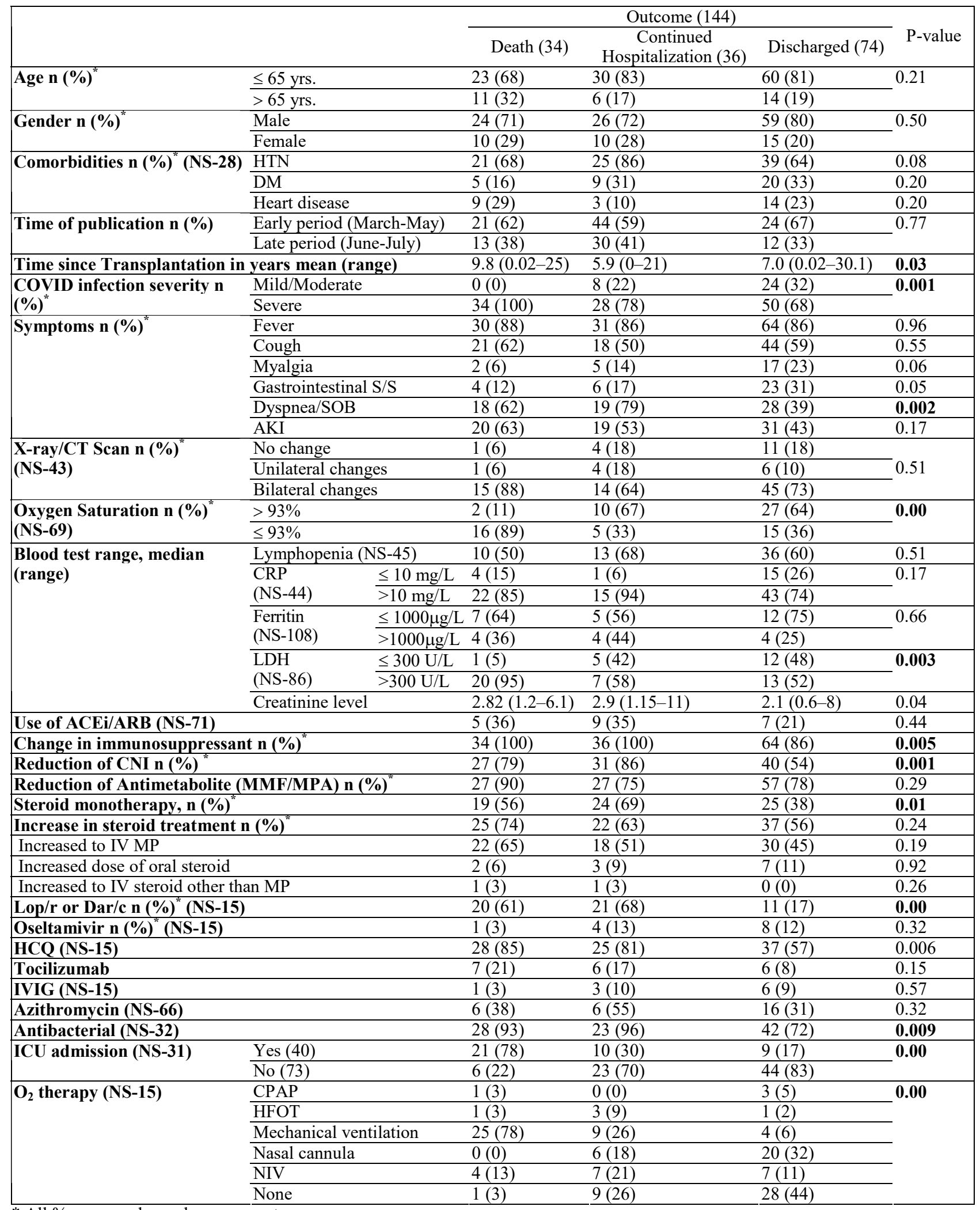

* All \% expressed as column percentage

NS - Not specified; HTN - Hypertension; DM - Diabetes mellitus; SOB: Shortness of breath; CRP - C reactive protein; LDH - Lactate dehydrogenase; IL 6 - Interleukin 6; CPAP: Continuous positive airway pressure; HFOT - High flow oxygen therapy; NIV - Noninvasive ventilation; CNI: Calcineurin inhibitor; MMF - Mycophenolate Mofetil; MPA - Mycophenolic Acid; MP: Methyl prednisone; Lop/r Lopinavir/ritonavir; Dar/c - Darunavir/cobicistat; HCQ - Hydroxychloroquine; IVIG - Intravenous Immunoglobulin 


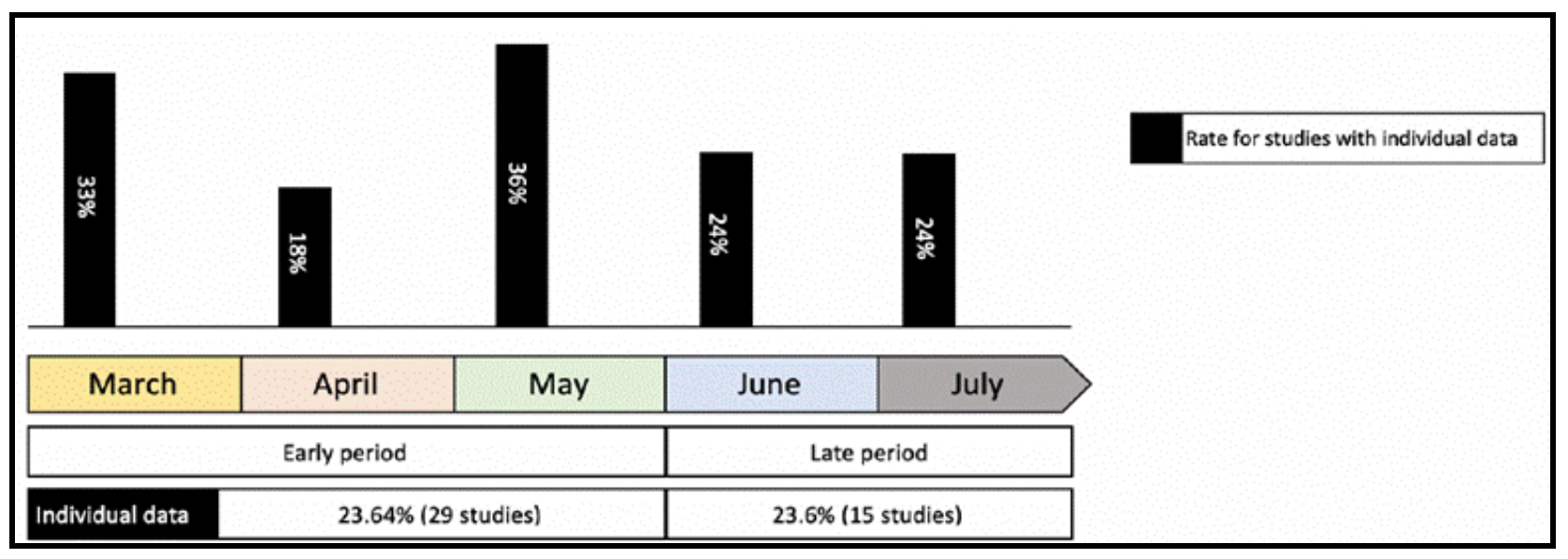

Figure 2. Mortality rate based on snapshot of time period captured by the studies

Table 6

Difference in survival based on therapeutic factors

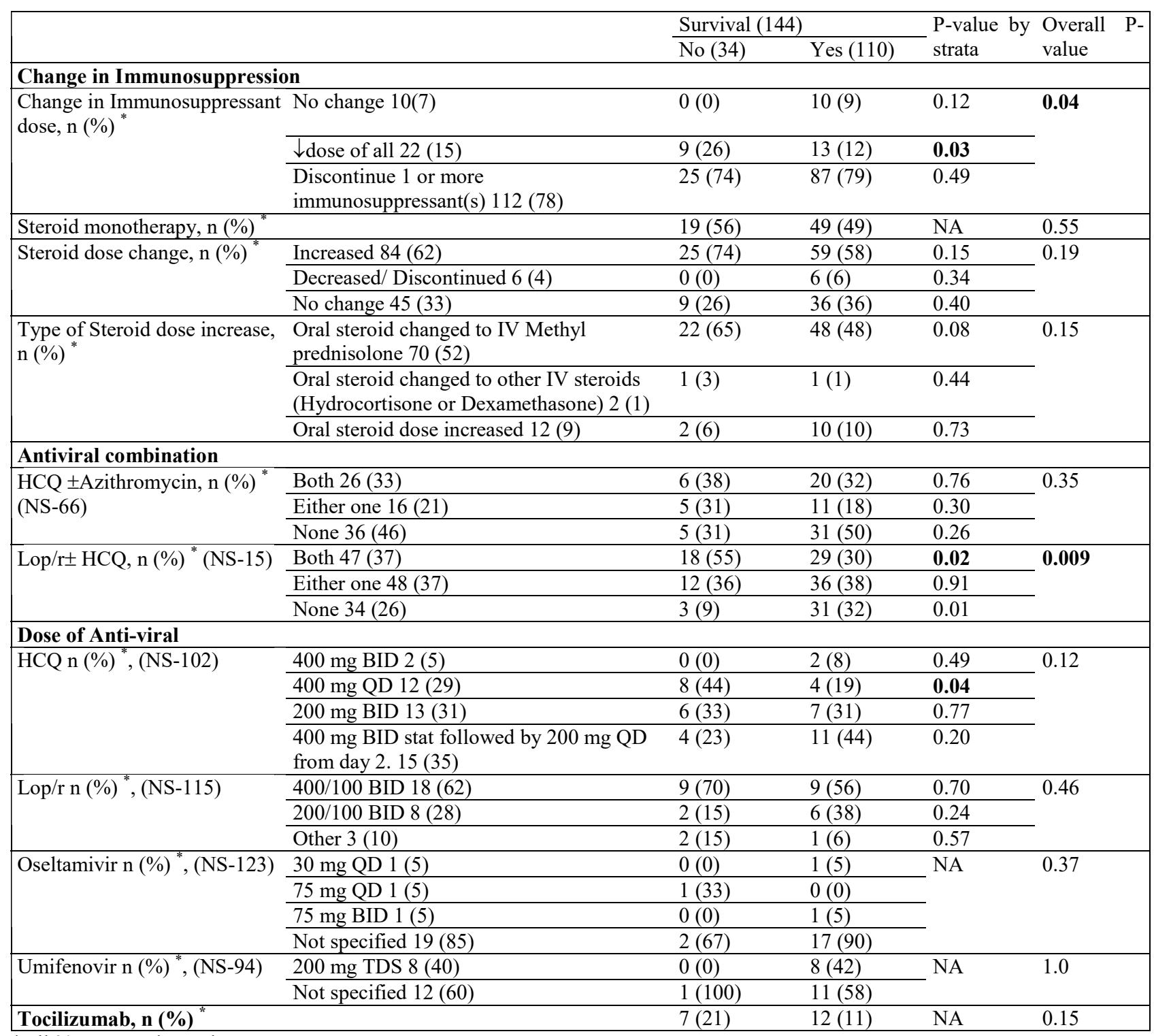

*All \% expressed as column percentage

NS - Not specified; NA - Not applicable; Lop/r - Lopinavir/ritonavir; HCQ - Hydroxychloroquine. 


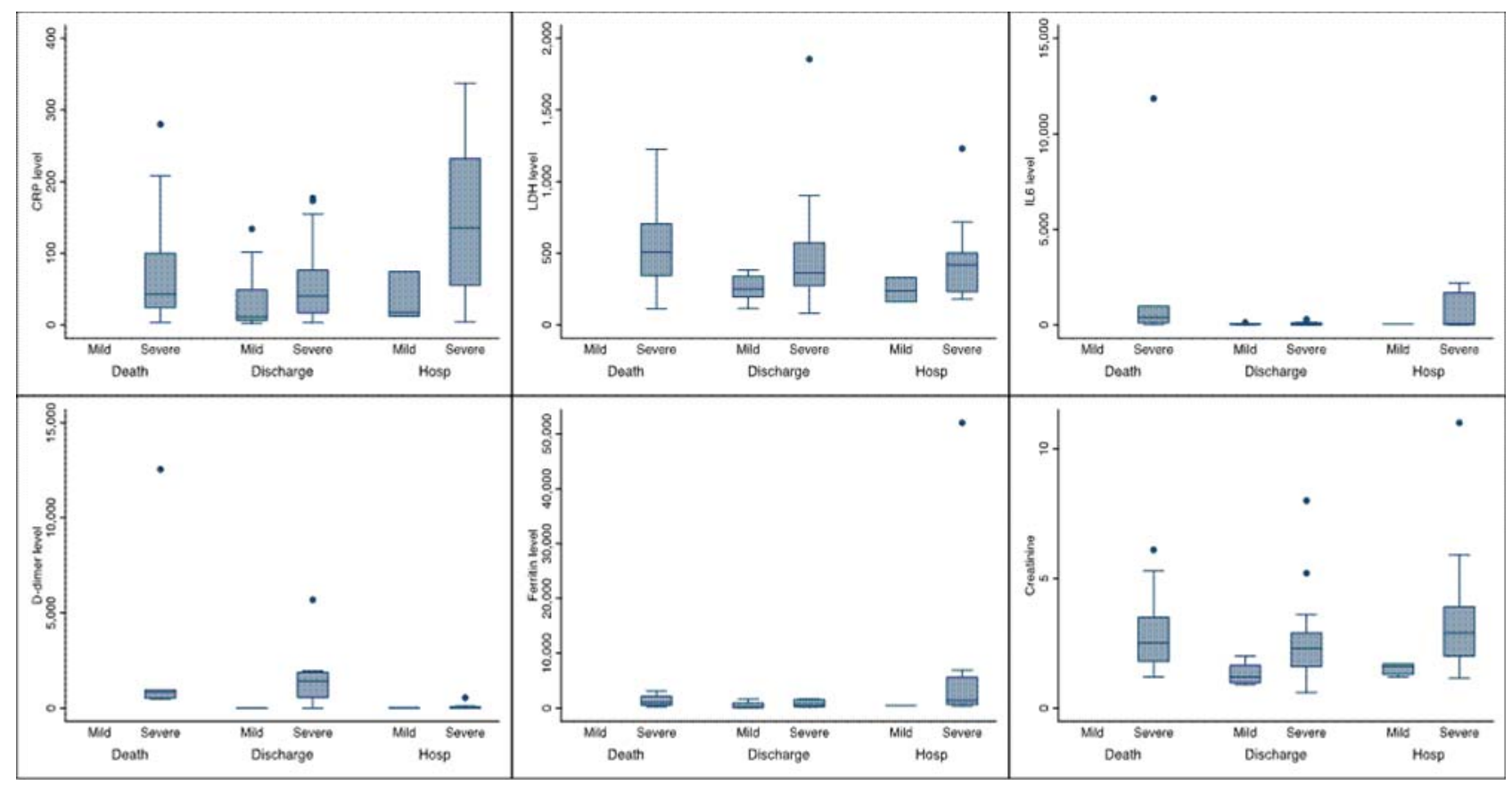

Figure 3. Box plot for immunological markers and serum creatinine stratified by disease severity and outcome for COVID-19 in renal transplant recipients. Boxes and whiskers represent the distribution of immunological markers and serum creatinine and the dots are the values greater than the third quartile and are considered outliers.

Introducere. COVID-19 reprezintă o provocare pentru pacienții candidați pentru transplant renal.

Metode. A fost realizată o sinteză sistematică ce a evaluat infecția COVID-19 la pacienții transplantați renal. Caracteristicile clinice, de laborator, radiologice, modificările terapiei imunosupresoare au fost analizate. Severitatea COVID-19 a fost clasificată în ușoară, moderată şi severă. Efectul urmărit a fost evoluţia pacientului externare, spitalizare sau deces.

Rezultate. 44 de articole cu date individuale și 13 articole cu date multiple de la 149, respectiv 561 de pacienți cu transplant renal și infecție COVID-19 din Asia, Europa și America au intrunit criteriile de includere. $76 \%$ dintre pacienți au avut boală severă. Pacienții cu boală severă au avut niveluri mai mari ale CRP, LDH, feritinei și D-Dimeri, precum și afectare pulmonară bilaterală la momentul internării. Genul, vârsta și comorbiditățile nu au influențat severitatea bolii. Pacienții cu boală severă au avut o reducere progresivă a CNI și au necesitat mai multe tratamente antivirale. Efectul a fost raportat la 145 de cazuri dintre care 34 (23\%) au decedat. Durata mai mare de la transplant, hipoxia și niveluri mai mari ale LDH s-au asociat cu mortalitatea. Strategiile de reducere ale imunosupresiei, nivelurile mari ale cortcoizilor administraţi parenteral și combinațiile de antivirale nu au fost un avantaj. Observații similare au fost făcute în studiile altor cazuri.

Concluzii. Infecția COVID-19 este asociată cu o rată mare a severității bolii și a mortalității. Nivelurile ridicate ale $L D H$ și durata mai mare de la transplant au prezis severitatea și mortalitatea bolii. Niciun tratament impotriva COVID-19 nu s-a asociat cu o îmbunătățire a prognosticului bolii la pacienții transplantați renal.

Correspondence to: Hani M Wadei, MD, Associate Professor of Medicine, Department of Transplantation, Department of Medicine, Division of Nephrology and Hypertension,

Mayo Clinic, 4500 San Pablo Rd. Jacksonville, FL 32224

Phone: 9049563259

E-mail: wadei.hani@mayo.edu 
Acknowledgements: None

Conflict of interest disclosure: The authors declare no conflict of interest

\section{REFERENCES}

1. WHO. Naming the coronavirus disease (COVID-19) and the virus that causes it. 2020; Available from: https://www.who.int/ emergencies/diseases/novel-coronavirus-2019/technical-guidance/naming-the-coronavirus-disease-(covid-2019)-and-the-virus-thatcauses-it.

2. Heymann, D.L. and N. Shindo, COVID-19: what is next for public health? Lancet, 2020. 395(10224): p. 542-545.

3. Mehta, P., D.F. McAuley, M. Brown, E. Sanchez, R.S. Tattersall, and J.J. Manson, COVID-19: consider cytokine storm syndromes and immunosuppression. Lancet, 2020. 395(10229): p. 1033-1034.

4. NIH. New coronavirus stable for hours on surfaces. 2020; Available from: https://www.nih.gov/news-events/news-releases/newcoronavirus-stable-hours-surfaces.

5. Yuki, K., M. Fujiogi, and S. Koutsogiannaki, COVID-19 pathophysiology: A review. Clin Immunol, 2020. 215: p. 108427.

6. Ruan, Q., K. Yang, W. Wang, L. Jiang, and J. Song, Clinical predictors of mortality due to COVID-19 based on an analysis of data of 150 patients from Wuhan, China. Intensive Care Med, 2020. 46(5): p. 846-848.

7. Xu, Z., L. Shi, Y. Wang, J. Zhang, L. Huang, C. Zhang, et al., Pathological findings of COVID-19 associated with acute respiratory distress syndrome. Lancet Respir Med, 2020. 8(4): p. 420-422.

8. WHO. Clinical management of severe acute respiratory infection(SARI) when COVID-19 disease is suspected. 2020; Available from: https://apps.who.int/iris/bitstream/handle/10665/331446/WHO-2019-nCoV-clinical-2020.4-eng.pdf? sequence = 1\&isAllowed = y.

9. Peng, F., L. Tu, Y. Yan, P. Hu, R. Wang, Q. Hu, et al., Management and Treatment of COVID-19: The Chinese Experience. Can J Cardiol, 2020.

10. PRISMA. PRISMA flow diagram. 2015; Available from: http://prisma-statement.org/PRISMAStatement/FlowDiagram.

11. Cochrane. General principles for dealing with missing data. 2020; Available from: https://handbook-5-1.cochrane.org/chapter 16/16_1_2_general_principles_for_dealing_with_missing_data.htm.

12. Machado, D.J.B. and L.E. Ianhez, COVID-19 pneumonia in kidney transplant recipients-Where we are? Transpl Infect Dis, 2020: p. e13306.

13. Hsu, J.J., P. Gaynor, M. Kamath, A. Fan, F. Al-Saffar, D. Cruz, et al., COVID-19 in a High-Risk Dual Heart and Kidney Transplant Recipient. Am J Transplant, 2020.

14. Patel, U., P. Malik, M.S. Usman, D. Mehta, A. Sharma, F.A. Malik, et al., Age-Adjusted Risk Factors Associated with Mortality and Mechanical Ventilation Utilization Amongst COVID-19 Hospitalizations-a Systematic Review and Meta-Analysis. SN Compr Clin Med, 2020: p. 1-10.

15. Fedson, D.S., S.M. Opal, and O.M. Rordam, Hiding in Plain Sight: an Approach to Treating Patients with Severe COVID-19 Infection. mBio, 2020. 11(2).

16. Cao, B., Y. Wang, D. Wen, W. Liu, J. Wang, G. Fan, et al., A Trial of Lopinavir-Ritonavir in Adults Hospitalized with Severe Covid-19. N Engl J Med, 2020. 382(19): p. 1787-1799.

17. Jin, J.M., P. Bai, W. He, F. Wu, X.F. Liu, D.M. Han, et al., Gender Differences in Patients With COVID-19: Focus on Severity and Mortality. Front Public Health, 2020. 8: p. 152.

18. Guan, W.J., W.H. Liang, Y. Zhao, H.R. Liang, Z.S. Chen, Y.M. Li, et al., Comorbidity and its impact on 1590 patients with COVID-19 in China: a nationwide analysis. Eur Respir J, 2020. 55(5).

19. Zhou, F., T. Yu, R. Du, G. Fan, Y. Liu, Z. Liu, et al., Clinical course and risk factors for mortality of adult inpatients with COVID-19 in Wuhan, China: a retrospective cohort study. Lancet, 2020. 395(10229): p. 1054-1062.

20. Omer, S.B., P. Malani, and C. del Rio, The COVID-19 Pandemic in the US: A Clinical Update. JAMA, 2020. 323(18): p. $1767-1768$.

21. Basu, A., Estimating The Infection Fatality Rate Among Symptomatic COVID-19 Cases In The United States. Health Aff (Millwood), 2020: p. 101377hlthaff202000455.

22. Buckner, F.S., D.J. McCulloch, V. Atluri, M. Blain, S.A. McGuffin, A.K. Nalla, et al., Clinical Features and Outcomes of 105 Hospitalized patients with COVID-19 in Seattle, Washington. Clin Infect Dis, 2020.

23. Robbins-Juarez, S.Y., L. Qian, K.L. King, J.S. Stevens, S.A. Husain, J. Radhakrishnan, et al., Outcomes for Patients With COVID-19 and Acute Kidney Injury: A Systematic Review and Meta-Analysis. Kidney International Reports, 2020.

24. AlGhamdi, M., F. Mushtaq, N. Awn, and S. Shalhoub, MERS CoV infection in two renal transplant recipients: case report. Am J Transplant, 2015. 15(4): p. 1101-4.

25. Abrishami, A., S. Samavat, B. Behnam, M. Arab-Ahmadi, M. Nafar, and M. Sanei Taheri, Clinical Course, Imaging Features, and Outcomes of COVID-19 in Kidney Transplant Recipients. Eur Urol, 2020.

26. Ahmad, S.H., R. Smith, and B. Camilleri, Belatacept, kidney transplantation and COVID-19: Successful management of the first reported case within the United Kingdom. Clin Transplant, 2020: p. e14026.

27. Alberici, F., E. Delbarba, C. Manenti, L. Econimo, F. Valerio, A. Pola, et al., A single center observational study of the clinical characteristics and short-term outcome of 20 kidney transplant patients admitted for SARS-CoV2 pneumonia. Kidney Int, 2020. 97(6): p. 1083-1088.

28. Arpali, E., B. Akyollu, B. Yelken, S. Tekin, A. Turkmen, and B. Kocak, Case report: A kidney transplant patient with mild COVID-19. Transpl Infect Dis, 2020: p. e13296.

29. Banerjee, D., J. Popoola, S. Shah, I.C. Ster, V. Quan, and M. Phanish, COVID-19 infection in kidney transplant recipients. Kidney Int, 2020. 97(6): p. 1076-1082. 
30. Billah, M., A. Santeusanio, V. Delaney, P. Cravedi, and S.S. Farouk, A catabolic state in a kidney transplant recipient with COVID-19. Transpl Int, 2020.

31. Bussalino, E., A. De Maria, R. Russo, and E. Paoletti, Immunosuppressive therapy maintenance in a kidney transplant recipient with SARS-CoV-2 pneumonia: A case report. Am J Transplant, 2020.

32. Chen, S., Q. Yin, H. Shi, D. Du, S. Chang, L. Ni, et al., A familial cluster, including a kidney transplant recipient, of Coronavirus Disease 2019 (COVID-19) in Wuhan, China. Am J Transplant, 2020.

33. Chen, D., B. Yang, Y. Zhang, L. Chen, L. Wei, W. Zhang, et al., Withdrawing mycophenolate mofetil in treating a young kidney transplant recipient with COVID-19: A case report. Medicine (Baltimore), 2020. 99(24): p. e20481.

34. Cheng, D.R., J.Q. Wen, Z.Z. Liu, T.F. Lv, and J.S. Chen, Coronavirus disease 2019 in renal transplant recipients: report of two cases. Transpl Infect Dis, 2020: p. e13329.

35. Dirim, A.B., E. Demir, A.R. Ucar, N. Garayeva, S. Safak, O.A. Oto, et al., Fatal SARS-CoV-2 infection in a renal transplant recipient. CEN Case Rep, 2020: p. 1-4.

36. Faguer, S., A. Del Bello, F. Abravanel, M.-L. Nicolau-Travers, and N. Kamar, Tocilizumab for Hemophagocytic Syndrome in a Kidney Transplant Recipient With COVID-19. Annals of Internal Medicine, 2020.

37. Fontana, F., G. Alfano, G. Mori, A. Amurri, L. Tei, M. Ballestri, et al., COVID-19 pneumonia in a kidney transplant recipient successfully treated with tocilizumab and hydroxychloroquine. Am J Transplant, 2020.

38. Fung, M., C.Y. Chiu, C. DeVoe, S.B. Doernberg, B.S. Schwartz, C. Langelier, et al., Clinical outcomes and serologic response in solid organ transplant recipients with COVID-19: A case series from the United States. Am J Transplant, 2020.

39. Gandolfini, I., M. Delsante, E. Fiaccadori, G. Zaza, L. Manenti, A. Degli Antoni, et al., COVID-19 in kidney transplant recipients. Am J Transplant, 2020.

40. Guillen, E., G.J. Pineiro, I. Revuelta, D. Rodriguez, M. Bodro, A. Moreno, et al., Case report of COVID-19 in a kidney transplant recipient: Does immunosuppression alter the clinical presentation? Am J Transplant, 2020.

41. Huang, J., H. Lin, Y. Wu, Y. Fang, R. Kumar, G. Chen, et al., COVID-19 in posttransplant patients-report of 2 cases. Am J Transplant, 2020.

42. Jiang, J., Y. Miao, Y. Zhao, X. Lu, P. Zhou, X. Zhou, et al., Convalescent plasma therapy: Helpful treatment of COVID-19 in a kidney transplant recipient presenting with serve clinical manifestation and complex complications. Clin Transplant, 2020: p. e14025.

43. Johnson, K.M., J.J. Belfer, G.R. Peterson, M.R. Boelkins, and L.E. Dumkow, Managing COVID-19 in Renal Transplant Recipients: A Review of Recent Literature and Case Supporting Corticosteroid-sparing Immunosuppression. Pharmacotherapy, 2020.

44. Kates, O.S., C.E. Fisher, H.C. Stankiewicz-Karita, A.K. Shepherd, E.C. Church, S.G. Kapnadak, et al., Earliest cases of coronavirus disease 2019 (COVID-19) identified in solid organ transplant recipients in the United States. American Journal of Transplantation, 2020.

45. Kim, Y., O. Kwon, J.H. Paek, W.Y. Park, K. Jin, M. Hyun, et al., Two distinct cases with COVID-19 in kidney transplant recipients. Am J Transplant, 2020.

46. Kocak, B., E. Arpali, B. Akyollu, B. Yelken, S. Tekin, M. Kanbay, et al., A Case Report of Oligosymptomatic Kidney Transplant Patients with COVID-19: Do They Pose a Risk to Other Recipients? Transplant Proc, 2020.

47. Kolonko, A., S. Dudzicz, A. Wiecek, and R. Król, COVID-19 infection in solid organ transplant recipients: A single-center experience with patients immediately after transplantation. Transpl Infect Dis, 2020: p. e13381.

48. Maritati, F., E. Cerutti, L. Zuccatosta, A. Fiorentini, C. Finale, M. Ficosecco, et al., SARS-CoV-2 infection in kidney transplant recipients: experience of the Italian marche region. Transpl Infect Dis, 2020: p. e13377.

49. Marx, D., B. Moulin, S. Fafi-Kremer, I. Benotmane, G. Gautier, P. Perrin, et al., First case of COVID-19 in a kidney transplant recipient treated with belatacept. Am J Transplant, 2020.

50. Mella, A., S. Mingozzi, E. Gallo, A. Lavacca, M. Rossetti, R. Clari, et al., Case series of six kidney transplanted patients with COVID-19 pneumonia treated with tocilizumab. Transpl Infect Dis, 2020: p. e13348.

51. Meziyerh, S., T.C. Zwart, R.W. van Etten, J.A. Janson, T. van Gelder, I.P.J. Alwayn, et al., Severe COVID-19 in a renal transplant recipient: A focus on pharmacokinetics. Am J Transplant, 2020.

52. Mohan, S., Early Description of Coronavirus 2019 Disease in Kidney Transplant Recipients in New York. J Am Soc Nephrol, 2020.

53. Nair, V., N. Jandovitz, J.S. Hirsch, G. Nair, M. Abate, M. Bhaskaran, et al., COVID-19 in kidney transplant recipients. American Journal of Transplantation, 2020.

54. Namazee, N., H. Mahmoudi, P. Afzal, and S. Ghaffari, Novel Corona Virus 2019 pneumonia in a kidney transplant recipient. American Journal of Transplantation, 2020.

55. Ning, L., L. Liu, W. Li, H. Liu, J. Wang, Z. Yao, et al., Novel coronavirus (SARS-CoV-2) infection in a renal transplant recipient: Case report. Am J Transplant, 2020.

56. Fernández-Ruiz, M., A. Andrés, C. Loinaz, J.F. Delgado, F. López-Medrano, R. San Juan, et al., COVID-19 in solid organ transplant recipients: A single-center case series from Spain. Am J Transplant, 2020.

57. Seminari, E., M. Colaneri, M. Sambo, I. Gallazzi, A. Di Matteo, S. Roda, et al., SARS Cov-2 infection in a renal-transplanted patient: A case report. Am J Transplant, 2020.

58. Shingare, A., M.M. Bahadur, and S. Raina, COVID-19 in recent kidney transplant recipients. Am J Transplant, 2020.

59. Silva, F., A. Cipriano, H. Cruz, J. Tavares, J. Fragoso, J. Malheiro, et al., SARS-CoV-2 infection in kidney transplant recipients: Early report of five cases. Transpl Infect Dis, 2020: p. e13394.

60. Thammathiwat, T., S. Tungsanga, K. Tiankanon, P. Torvorapanit, W. Chumpangern, S. Udomkarnjananun, et al., A case of successful treatment of severe COVID-19 pneumonia with favipiravir and tocilizumab in post-kidney transplant recipient. Transpl Infect Dis, 2020: p. e13388.

61. Wang, J., X. Li, G. Cao, X. Wu, Z. Wang, and T. Yan, COVID-19 in a Kidney Transplant Patient. Eur Urol, 2020. 77(6): p. 769-770.

62. Zhang, H., Y. Chen, Q. Yuan, Q.X. Xia, X.P. Zeng, J.T. Peng, et al., Identification of Kidney Transplant Recipients with Coronavirus Disease 2019. Eur Urol, 2020. 77(6): p. 742-747. 
63. Zhang, M., J. Zhang, H. Shi, B. Liu, and F. Zeng, Viral Shedding Prolongation in a Kidney Transplant Patient with COVID-19 Pneumonia. Am J Transplant, 2020.

64. Zhong, Z., Q. Zhang, H. Xia, A. Wang, W. Liang, W. Zhou, et al., Clinical characteristics and immunosuppressant management of coronavirus disease 2019 in solid organ transplant recipients. Am J Transplant, 2020.

65. Zhu, L., N. Gong, B. Liu, X. Lu, D. Chen, S. Chen, et al., Coronavirus Disease 2019 Pneumonia in Immunosuppressed Renal Transplant Recipients: A Summary of 10 Confirmed Cases in Wuhan, China. Eur Urol, 2020. 77(6): p. 748-754.

66. Zhu, L., X. Xu, K. Ma, J. Yang, H. Guan, S. Chen, et al., Successful recovery of COVID-19 pneumonia in a renal transplant recipient with long-term immunosuppression. Am J Transplant, 2020.

67. Akalin, E., Y. Azzi, R. Bartash, H. Seethamraju, M. Parides, V. Hemmige, et al., Covid-19 and Kidney Transplantation. N Engl J Med, 2020. 382(25): p. 2475-2477.

68. Montagud-Marrahi, E., F. Cofan, J.V. Torregrosa, D. Cucchiari, P. Ventura-Aguiar, I. Revuelta, et al., Preliminary data on outcomes of SARS-CoV-2 infection in a Spanish single center cohort of kidney recipients. Am J Transplant, 2020.

69. Husain, S.A., G. Dube, H. Morris, H. Fernandez, J.H. Chang, K. Paget, et al., Early Outcomes of Outpatient Management of Kidney Transplant Recipients with Coronavirus Disease 2019. Clin J Am Soc Nephrol, 2020.

70. Crespo, M., M.J. Pérez-Sáez, D. Redondo-Pachón, L. Llinàs-Mallol, M.M. Montero, J. Villar-García, et al., COVID-19 in elderly kidney transplant recipients. Am J Transplant, 2020.

71. Pascual, J., E. Melilli, C. Jiménez-Martín, E. González-Monte, S. Zárraga, A. Gutiérrez-Dalmau, et al., COVID-19-related Mortality During the First 60 Days After Kidney Transplantation. Eur Urol, 2020.

72. Bossini, N., F. Alberici, E. Delbarba, F. Valerio, C. Manenti, S. Possenti, et al., Kidney transplant patients with SARS-CoV-2 infection: the brescia renal COVID task force experience. Am J Transplant, 2020.

73. Cravedi, P., S.M. Suraj, Y. Azzi, M. Haverly, S. Farouk, M.J. Pérez-Sáez, et al., COVID-19 and Kidney Transplantation: Results from the TANGO International Transplant Consortium. Am J Transplant, 2020.

74. Ghaffari Rahbar, M., M. Nafar, A. Khoshdel, N. Dalili, A. Abrishami, A. Firouzan, et al., Low rate of COVID-19 pneumonia in kidney transplant recipients - A battle between infection and immune response? Transpl Infect Dis, 2020: p. e13406.

75. Pérez-Sáez, M.J., M. Blasco, D. Redondo-Pachón, P. Ventura Aguilar, T. Bada-Bosch, I. Pérez-Flores, et al., Use of tocilizumab in kidney transplant recipients with COVID-19. Am J Transplant, 2020.

76. Demir, E., M. Uyar, E. Parmaksiz, A. Sinangil, B. Yelken, A.B. Dirim, et al., COVID-19 in kidney transplant recipients: A multicenter experience in Istanbul. Transpl Infect Dis, 2020: p. e13371.

77. Lubetzky, M., M.J. Aull, R. Craig-Schapiro, J.R. Lee, J. Marku-Podvorica, T. Salinas, et al., Kidney allograft recipients, immunosuppression, and coronavirus disease-2019: a report of consecutive cases from a New York City transplant center. Nephrol Dial Transplant, 2020.

78. Monfared, A., S. Dashti-Khavidaki, R. Jafari, A. Jafari, E. Ramezanzade, M.K. Lebadi, et al., Clinical Characteristics and Outcome of Covid-19 Pneumonia in Kidney Transplant Recipients in Razi hospital, Rasht, Iran. Transpl Infect Dis, 2020: p. e13420.

Received $12^{\text {th }}$ October 2020 